\title{
Complete Band Gaps in Nano-Piezoelectric Phononic Crystals
}

\author{
Edson Jansen Pedrosa de Miranda Jra ${ }^{a, b *}$, José Maria Campos Dos Santos ${ }^{a}$ \\ ${ }^{a}$ Departamento de Mecânica Computacional - DMC, Universidade Estadual de Campinas - UNICAMP, \\ Rua Mendeleyev, 200, CEP 13083-970, Campinas, SP, Brasil. \\ ${ }^{b}$ Departamento de Educação Profissional - DEP, Instituto Federal de Educação, Ciência e Tecnologia \\ do Maranhão - IFMA, Rua Afonso Pena, 174, CEP 65010-030, São Luís, MA, Brasil.
}

Received: March 17, 2017; Revised: August 16, 2017; Accepted: September 17, 2017

\begin{abstract}
We study the band structure of elastic waves propagating in a nano-piezoelectric phononic crystal consisting of a polymeric matrix reinforced by $\mathrm{BaTiO}_{3}$ inclusions in square, rectangular, triangular, honeycomb and Kagomé lattices. We also investigate the influence of inclusion cross section geometry circular, hollow circular, square and rotated square with a $45^{\circ}$ angle of rotation with respect to $x$ and $y$ axes. Plane wave expansion method is used to solve the governing equations of motion of a piezoelectric solid based on classical elasticity theory, ignoring nanoscopic size effects, considering two-dimensional periodicity and wave propagation in the $x y$ plane. Complete band gaps between XY and $\mathrm{Z}$ modes are observed for all inclusions and the best performance is for circular inclusion in a triangular lattice. Piezoelectricity influences significantly the band gaps for hollow circular inclusion in lower frequencies. We suggest that nano-piezoelectric phononic crystals are feasible for elastic vibration management in GHz.
\end{abstract}

Keywords: nano-piezoelectric phononic crystal, band structure, plane wave expansion method, complete band gaps, vibration control.

\section{Introduction}

Phononic crystals (PCs) are artificial periodic composites designed to exhibit phononic band gaps and they have been quite studied ${ }^{1-14}$. There are no mechanical (elastic or acoustic) propagating waves in phononic band gaps, only evanescent waves. These band gaps are created by the periodically mismatch between the constituent materials. This mismatch can be considered to arise either from difference of material properties or geometry (continuum-scale theory), or from interatomic force constants and masses (atomic-scale theory).

The ability of creating phononic band gaps is similar to the electronic and photonic band gaps in semiconductors and photonic crystals ${ }^{15-16}$, respectively. The physical origin of phononic and photonic band gaps can be understood at micro-scale using the classical wave theory to describe the Bragg and Mie resonances, respectively, based on the scattering of mechanical and electromagnetic waves propagating within the crystal ${ }^{17}$.

PCs have many applications, such as vibration isolation technology ${ }^{18-22}$, acoustic barriers/filters ${ }^{23-25}$, noise suppression devices $^{26-27}$, surface acoustic devices ${ }^{28}$, architectural design ${ }^{29}$, sound shields ${ }^{30}$, acoustic diodes ${ }^{31}$, elastic metamaterials ${ }^{21-22,25,27,32}$ and thermal metamaterials $\mathrm{s}^{33-39}$.

There are also smart PCs that have been studied, such as piezoelectric ${ }^{40-54}$, piezomagnetic ${ }^{55-58}$ and magnetoelectroelastic ${ }^{14,59-63}$ PCs. Among these intelligent PCs, piezoelectric PCs are sensitive to elastic and electric field. Even though the band structure behavior of piezoelectric PCs have been already investigated, to our knowledge only few studies ${ }^{46-49,51}$ focused on the influence of inclusion geometry and lattice on band gap formation. Wang and co-workers ${ }^{46}$ considered a piezoelectric $\mathrm{PC}$ with square lattice and different inclusion geometries (regular triangle, square, hexagon, circle and oval). They found that the largest complete band gap is obtained by selecting the inclusion with the same symmetry of lattice for the first band gap. Hsu et al. ${ }^{47}$ studied the band structure of a piezoelectric PC with square and triangular lattices using Mindlin-Reissner plate theory and considering only circular inclusion. Qian and co-workers ${ }^{48}$ studied the band structure of piezoelectric PCs with square lattice and circular and square inclusions. They revealed the existence of several very large complete band gaps in PZT rods reinforced polythene composite. Zhou et al..$^{49}$ investigated the band structure of piezoelectric PCs consisting of rectangular inclusion in an epoxy substrate. They considered a PC with square and triangular lattices. Wang and co-workers ${ }^{51}$ considered a piezoelectric PC with circular inclusions and square and rectangular lattices. They verified that the largest band gap width is not always obtained for a square lattice. In some cases, rectangular lattice can produce broader gaps. In this context, we extend the studies about piezoelectric $\mathrm{PCs}^{40-54}$ considering the influence of different inclusion geometries - circular, hollow circular, square and rotated square with $45^{\circ}$ angle of rotation with respect to the $x$ and $y$ axes, and different lattices - square, rectangular, triangular, honeycomb and Kagomé on the band structure. 
Furthermore, researches have used PCs on the scale $\mu \mathrm{m}^{10,17,64,65}$ and $\mathrm{mm}$, resulting in band gaps ranging from $\mathrm{GHz}$ and $\mathrm{kHz}$ to $\mathrm{MHz}$, respectively. More recently, with the advance of nanomaterials fabrication, nanophononic crystals have been studied and it is possible to control wave propagation in a frequency range from hypersonic ${ }^{3-6,66-75}$ to thermal ${ }^{33-39}$. However, nano-piezoelectric PCs have not been investigated yet even though studies about nano-piezoelectric materials have been reported $\mathrm{d}^{76-79}$.

The main purpose of this study is to investigate the elastic band structure, also known as dispersion relation, of a nano-piezoelectric phononic crystal with square, rectangular, triangular, honeycomb and Kagomé lattices, composed by a polymeric matrix and $\mathrm{BaTiO}_{3}$ inclusions, considering wave propagation in the $x y$ plane and two-dimensional periodicity. We consider the following inclusions geometries - circular, hollow circular, square and rotated square with a $45^{\circ}$ angle of rotation with respect to the $x$ and $y$ axes. To obtain the elastic band structure we use the semi-analytical plane wave expansion $^{1,2}$ (PWE) method.

To the best of our knowledge, it is the first time that the band structure of nano-piezoelectric PCs with different inclusion geometries and different lattices is investigated.

\section{2D Nano-Piezoelectric Phononic Crystal Model by PWE Method}

PWE formulation of a 2D PC is presented in this section. PWE method ${ }^{1,2}$, also known as $\omega(\mathbf{k})$ method, is one of the most used methods to calculate the elastic band structure of PCs and it has been applied in micro ${ }^{10}$ and nanophononic crystals $^{71,75}$ (NPCs). PWE method is used to solve the constitutive equations of a piezoelectric material based on classical elasticity theory, ignoring nanoscopic size effects, similar to other studies ${ }^{10,71,75}$.

We consider two-dimensional periodicity, transversely isotropic elastic solid and wave propagation in the $x y$ plane. Complete band gaps between XY (longitudinal-transverse vibration) and $Z$ (transverse vibration) modes are observed for all inclusion geometry - circular, hollow circular, square and rotated square, considering square, rectangular, triangular, honeycomb and Kagomé lattices. Piezoelectric effect on the $\mathrm{Z}$ modes is also investigated.

\subsection{D Phononic Crystal Model}

Figure 1 (a-e) sketches the cross section of nanopiezoelectric PC unit cells taking in to account square, rectangular, triangular, honeycomb and Kagomé lattices, respectively, with arbitrary inclusion geometry. Figure 1 (f-h) represents the first Brillouin zone ${ }^{80}$ (FBZ) for square, rectangular, triangular, honeycomb and Kagomé lattices. Note that triangular, honeycomb and Kagomé lattices present the same FBZ shape (Figure 1 (h)). We consider four types of $\mathrm{BaTiO}_{3}$ inclusions: circular, hollow circular, square and rotated square with a $45^{\circ}$ angle of rotation with respect to the $x, y$ axes. We underline that only $\mathrm{BaTiO}_{3}$ inclusions are rotated for rotated square configuration, not the entire lattice.

In addition, we highlight that we consider $1 / 4$ of the FBZ as the actual first irreducible Brillouin zone ${ }^{80}$ (FIBZ) for square and rotated square inclusions in triangular, honeycomb and Kagomé lattices because the symmetries of phononic crystals are reduced since the phononic crystal basis has different symmetry from the lattice ${ }^{81}$. Thus, for these configurations we scan $\mathrm{M}^{\prime}-\Gamma-\mathrm{X}-\mathrm{M}-\mathrm{X}^{\prime}-\mathrm{M}^{\prime}$. It is very important to make this adjustment since it changes the phononic crystal band structures ${ }^{81}$. For circular and hollow circular inclusions in triangular, honeycomb and Kagomé lattices, phononic crystal symmetry is not reduced thus we scan only $\mathrm{M}-\Gamma-\mathrm{X}-\mathrm{M}$.

FIBZ points in Figure $1(\mathrm{f}-\mathrm{h})$ are $\Gamma(0,0), \mathrm{X}\left(\frac{\pi}{a}, 0\right)$ and $\mathrm{M}\left(\frac{\pi}{a}, \frac{\pi}{a}\right)$ for square lattice, $\Gamma(0,0), \mathrm{X}\left(\frac{\pi}{a_{1}}, 0\right), \mathrm{M}\left(\frac{\pi}{a_{1}}, \frac{\pi}{a_{2}}\right)$ and $\mathrm{K}\left(0, \frac{\pi}{a_{2}}\right)$ for rectangular lattice, $\Gamma(0,0), \mathrm{X}\left(\frac{4 \pi}{3 a}, 0\right), \mathrm{M}$ $\left(\frac{\pi}{a}, \frac{\pi}{\sqrt{3 a}}\right), \mathrm{X}^{\prime}\left(\frac{2 \pi}{3 a}, \frac{2 \pi}{\sqrt{3 a}}\right)$ and $\mathrm{M}^{\prime}\left(0, \frac{2 \pi}{\sqrt{3 a}}\right)$ for triangular lattice, $\Gamma(0,0), \mathrm{X}\left(\frac{4 \pi}{3 \sqrt{3 a}}, 0\right), \mathrm{M}\left(\frac{\pi}{\sqrt{3 a}}, \frac{\pi}{3 a}\right), \mathrm{X}^{\prime}\left(\frac{2 \pi \sqrt{3}}{9 a}, \frac{2 \pi}{3 a}\right)$ and $\mathrm{M}^{\prime}$ $\left(0, \frac{2 \pi}{3 a}\right)$ for honeycomb lattice, and $\Gamma(0,0), \mathrm{X}\left(\frac{2 \pi}{3 a}, 0\right), \mathrm{M}$ $\left(\frac{\pi}{2 a}, \frac{\pi}{2 \sqrt{3 a}}\right), \mathrm{X}^{\prime}\left(\frac{\pi}{3 a}, \frac{\pi}{\sqrt{3 a}}\right)$ and $\mathrm{M}^{\prime}\left(0, \frac{\pi}{\sqrt{3 a}}\right)$ for Kagomé lattice, respectively, where $a$ is the lattice parameter for square, triangular, honeycomb and Kagomé lattices, whereas $a_{1}, a_{2}$ are the lattice parameters for rectangular lattice.

The constitutive equations of an elastic piezoelectric material are ${ }^{41}$ :

$$
\begin{gathered}
\sigma_{i j}=c_{i j k l} u_{k . l}-e_{l i j} E_{l}, \\
D_{i}=e_{i k l} u_{k . l}-\epsilon_{i l} E_{l},
\end{gathered}
$$

where $i, j, k, l=1,2,3, \sigma_{i j}$ is the elastic stress tensor, $D_{i}$ is the electric displacement vector, $u_{i}$ is the elastic displacement vector, $E_{l}$ is the electric field vector, $c_{i j k l}$ is the elastic stiffness tensor, $e_{l i j}$ is the piezoelectric tensor and $\epsilon_{i l}$ is the dielectric tensor. The standard tensor notation is used with Latin indices running from 1 to 3. They obey Einstein's summation convention when repeated.

We restricted the treatment to linear media, thus the elastic strain tensor $\varepsilon_{k l}$ is simplified:

$$
\varepsilon_{k l}=\frac{1}{2}\left(u_{k, l}+u_{l, k}\right) .
$$

Furthermore, based on the quasi-static approximation, there is no electro source (i.e. since there is no voltage source, this configuration is also known as an open circuit) and the curl is zero, thus the electric field is taken as gradient of scalar potential and one can write:

$$
E_{l}=-\phi_{, l}
$$


(a)

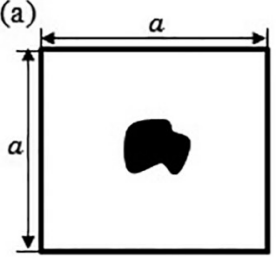

(f)

(b)
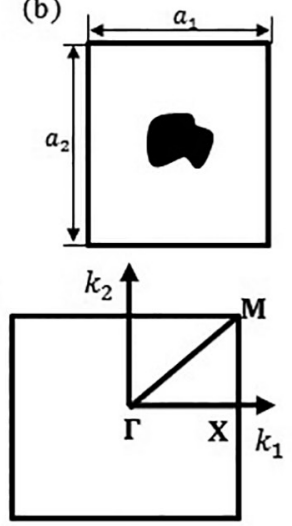

(c)

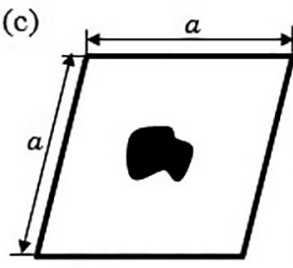

(g)

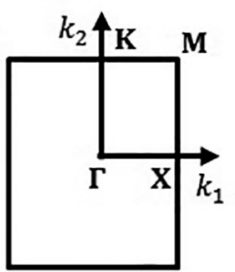

(d)
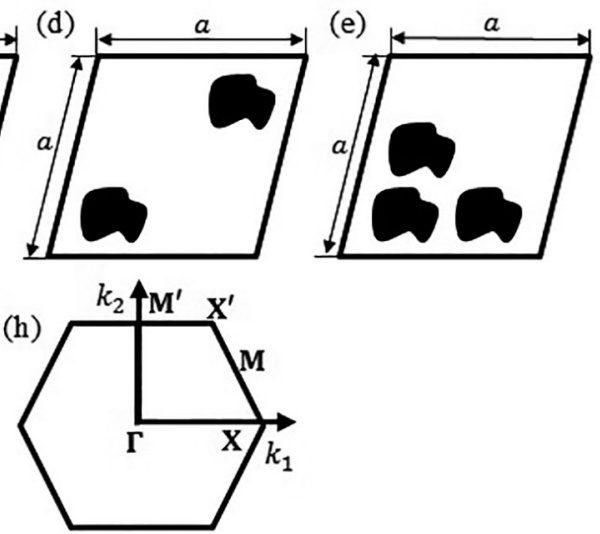

Figure 1. Transverse cross-section of the nano-piezoelectric phononic crystal unit cell: BaTiO inclusions distributed in a polymeric matrix for square (a), rectangular (b), triangular (c), honeycomb (d) and Kagomé (e) lattices. First Brillouin zone for square (f), rectangular (g), triangular (h), honeycomb $(\mathrm{h})$ and Kagomé $(\mathrm{h})$ lattices.

The differential equations of motion in absence of body forces are given by:

$$
\begin{gathered}
\sigma_{i j, i}=\rho \ddot{u}_{j}, \\
D_{i, i}=0,
\end{gathered}
$$

where $\rho$ is the mass density and dot denotes differentiation with respect to time. Substituting Eqs. (1-2) in Eqs. (5-6), applying the simplifications of Eqs. (3-4), considering a transversely isotropic elastic solid and for a two-dimensional problem, $\partial / \partial x_{3}=0$, results:

$$
\begin{aligned}
& \rho \ddot{u}_{1}=\left(c_{11} u_{1,1}+c_{12} u_{2,2}\right)_{, 1}+\left[c_{66}\left(u_{1,2}+u_{2,1}\right)\right]_{, 2}, \\
& \rho \ddot{u}_{2}=\left(c_{12} u_{1,1}+c_{11} u_{2,2}\right)_{, 2}+\left[c_{66}\left(u_{1,2}+u_{2,1}\right)\right]_{, 1}, \\
& \rho \ddot{u}_{3}=\left(c_{44} u_{3,1}+e_{15} \phi_{, 1}\right)_{, 1}+\left(c_{44} u_{3,2}+e_{15} \phi_{, 2}\right)_{, 2}, \\
& 0=\left(e_{15} u_{3,1}-\epsilon_{11} \phi_{, 1}\right)_{, 1}+\left(e_{15} u_{3,2}-\epsilon_{11} \phi_{, 2}\right)_{, 2},
\end{aligned}
$$

where $c_{66}=\frac{1}{2}\left(c_{11}-c_{12}\right)$. Note that Eqs. (7-10) are written using Voigt notation and from now on this notation is adopted.

In addition, considering a piezoelectric $\mathrm{PC}$, one can note that $c_{11}=c_{11}(\mathbf{r}), c_{12}=c_{12}(\mathbf{r}), c_{66}=c_{66}(\mathbf{r}), c_{44}=c_{44}(\mathbf{r})$, $e_{15}=e_{15}(\mathbf{r}), \epsilon_{11}=\epsilon_{11}(\mathbf{r}), \rho=\rho(\mathbf{r})$, because we consider two different materials $-\mathrm{BaTiO}_{3}$ inclusions and a polymeric matrix, and $u_{i}=u_{i}(\mathbf{r}, t)$. For a two-dimensional periodicity (the system has translational symmetry in $z$ direction and material parameters depend only on the $x$ and $y$ coordinates), then $\mathbf{r}=x \mathbf{e}_{1}+y \mathbf{e}_{2}(x, y \in \mathbb{R})$ is the two-dimensional spatial vector and $\mathbf{e}_{\mathrm{i}}(i=1,2)$ are the basis vectors in real space.
In order to eliminate the factor time in Eqs. (7-10), we apply the temporal Fourier transform. Applying FloquetBloch's theorem ${ }^{82-83}$, expanding Bloch wave amplitude as Fourier series in reciprocal space and considering wave propagation in the $x y$ plane $\left(k_{3}=0\right)$, we can write:

$$
\begin{aligned}
& u_{i}(\mathbf{r})=e^{j \mathbf{k} \mathbf{r}} u_{i \mathbf{k}}(\mathbf{r})=e^{j \mathbf{k} \cdot \mathbf{r}} \\
& \sum_{\mathbf{g}=-\infty}^{+\infty} u_{i \mathbf{k}}(\mathbf{g}) e^{j \mathbf{g} \cdot \mathbf{r}}= \\
& \sum_{\mathbf{g}=-\infty}^{+\infty} u_{i \mathbf{k}}(\mathbf{g}) e^{j(\mathbf{k}+\mathbf{g}) \cdot \mathbf{r}}
\end{aligned}
$$

where $u_{i \mathbf{k}}(\mathbf{r})$ is the Bloch wave amplitude, note that $u_{i \mathbf{k}}$ $(\mathbf{r})=u_{i \mathbf{k}}(\mathbf{r}+\overline{\mathbf{r}})$ and $u_{i}(\mathbf{r}+\overline{\mathbf{r}})=u_{i}(\mathbf{r}) e^{j \mathbf{k} \mathbf{r}}, e^{j \mathbf{k} \mathbf{r}}$ is called Bloch periodic boundary condition, $\mathbf{k}=\bar{u} \mathbf{b}_{1}+\bar{v} \mathbf{b}_{2}$ is the Bloch wave vector, $\bar{u}, \bar{v} \in \mathbb{Q}$ are the symmetry points within the FIBZ in reciprocal space, or we may write $\mathbf{k}=k_{1} \mathbf{e}_{1}+k_{2} \mathbf{e}_{2}, k_{1}, k_{2} \in \mathbb{R}$ are the point coordinates within the FIBZ in Figure 1 (f-h) for the reciprocal space. The basis vectors in reciprocal space $\mathbf{b}_{\mathrm{i}}(i=1,2)$ are defined as $\mathbf{a}_{\mathrm{i}} \cdot \mathbf{b}_{\mathrm{j}}=2 \pi \delta_{i j}, \delta_{i j}=0$ if $i \neq j$ or $\delta_{i j}$ $=1$ if $i=j$ is the Kronecker delta, $\mathbf{b}_{1}=2 \pi \frac{\mathbf{a}_{2} \times \mathbf{a}_{3}}{\mathbf{a}_{1} \cdot\left(\mathbf{a}_{2} \times \mathbf{a}_{3}\right)} \mathbf{b}_{2}=$ $2 \pi \frac{\mathbf{a}_{3} \times \mathbf{a}_{1}}{\mathbf{a}_{2} \cdot\left(\mathbf{a}_{3} \times \mathbf{a}_{1}\right)} \mathbf{a}_{\mathrm{i}}(i=1,2)$ are the components of the lattice vector $\overline{\mathbf{r}}=\left(\bar{p} \mathbf{a}_{1}+\bar{q} \mathbf{a}_{2}\right), \bar{p}, \bar{q} \in \mathbb{Z}$.

The lattice vector components are $\mathbf{a}_{\mathrm{i}}=a \mathbf{e}_{\mathrm{i}}(i=1,2)$ for square lattice, $\mathbf{a}_{1}=a_{1} \mathbf{e}_{1}, \mathbf{a}_{2}=a_{2} \mathbf{e}_{2}$ for rectangular lattice, $\mathbf{a}_{1}=$ $a \mathbf{e}_{1}, \mathbf{a}_{2}=\frac{a}{2} \mathbf{e}_{1}+\frac{a \sqrt{3}}{2} \mathbf{e}_{2}$ for triangular lattice, $\mathbf{a}_{1}=\frac{a \sqrt{3}}{2} \mathbf{e}_{1}$ $+\frac{3 a}{2} \mathbf{e}_{2}, \mathbf{a}_{2}=-\frac{a \sqrt{3}}{2} \mathbf{e}_{1}+\frac{3 a}{2} \mathbf{e}_{2}$ for honeycomb lattice and $\mathbf{a}_{1}=a \mathbf{e}_{1}+a \sqrt{3} \mathbf{e}_{2}, \mathbf{a}_{2}=-a \mathbf{e}_{1}+a \sqrt{3} \mathbf{e}_{2}$ for Kagomé lattice.

The reciprocal lattice vector is defined as $\mathrm{g}=\frac{2 \pi}{a}\left(m \mathbf{e}_{1}+n \mathbf{e}_{2}\right)$ $(m, n \in \mathbb{Z})$ for square lattice, $\mathbf{g}=2 \pi\left(\frac{m}{a_{1}} \mathbf{e}_{1}+\frac{n}{a_{2}} \mathbf{e}_{2}\right)$ for rectangular lattice, $\mathbf{g}=\frac{2 \pi}{a}\left[m \mathbf{e}_{1}+\frac{(-m+2 n)}{\sqrt{3}} \mathbf{e}_{2}\right]$ for triangular lattice, $\mathbf{g}=\frac{2 \pi}{a \sqrt{3}}\left[(m-n) \mathbf{e}_{1}+\frac{m+n}{\sqrt{3}} \mathbf{e}_{2}\right]$ for honeycomb lattice

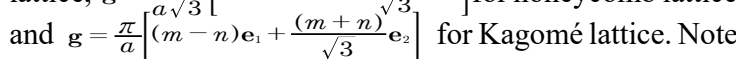
that $\mathbf{g}$ is a two-dimensional vector because we consider twodimensional periodicity.

Furthermore, we may expand $c_{11}, c_{12}, c_{66}, c_{44}, e_{15}, \epsilon_{11}, \rho$ in Fourier series on the reciprocal space as: 


$$
P(\mathbf{r})=\sum_{\overline{\mathbf{g}}=-\infty}^{+\infty} P(\overline{\mathbf{g}}) e^{j \overline{\mathbf{g}} \mathbf{.}},
$$

where $P$ is one of the $c_{11}, c_{12}, c_{66}, c_{44}, e_{15}, \epsilon_{11}, \rho$ and $\overline{\mathbf{g}}$ has the same expressions of $\mathbf{g}$ with $\bar{m}, \bar{n} \in \mathbb{Z}$. We use $\overline{\mathbf{g}}$ instead of $\mathbf{g}$ to highlight the difference between the Fourier series expansion of material properties and displacements.

Substituting Eqs. (11) and (12) in Eqs. (7-10), with $\tilde{\mathbf{g}}=$ $\overline{\mathbf{g}}+\mathbf{g}$, multiplying by $e^{-j \bar{g} . r}$ and integrating over the unit cell, we may write:

$$
\left(\mathbf{K}-\omega^{2} \mathbf{M}\right) \mathbf{q}=\mathbf{0}
$$

where

$$
\mathbf{K}=\left[\begin{array}{llll}
\mathbf{K}_{11} & \mathbf{K}_{12} & \mathbf{0} & \mathbf{0} \\
\mathbf{K}_{21} & \mathbf{K}_{22} & \mathbf{0} & \mathbf{0} \\
\mathbf{0} & \mathbf{0} & \mathbf{K}_{33} & \mathbf{K}_{34} \\
\mathbf{0} & \mathbf{0} & \mathbf{K}_{43} & \mathbf{K}_{44}
\end{array}\right]
$$

The sub-matrices in Eq. (14) are given by:

$$
\begin{aligned}
& \mathbf{K}_{11}=c_{11}(\overline{\mathbf{g}}-\mathbf{g})(\mathrm{k}+\mathrm{g})_{1}(\mathrm{k}+\overline{\mathrm{g}})_{1}+ \\
& c_{66}(\overline{\mathbf{g}}-\mathbf{g})(\mathrm{k}+\mathrm{g})_{2}(\mathrm{k}+\overline{\mathrm{g}})_{2}, \\
& \mathbf{K}_{12}=c_{12}(\overline{\mathbf{g}}-\mathbf{g})(\mathrm{k}+\mathrm{g})_{2}(\mathrm{k}+\overline{\mathrm{g}})_{1}+ \\
& c_{66}(\overline{\mathbf{g}}-\mathbf{g})(\mathrm{k}+\mathrm{g})_{1}(\mathrm{k}+\overline{\mathrm{g}})_{2}, \\
& \mathbf{K}_{21}=c_{12}(\overline{\mathbf{g}}-\mathbf{g})(\mathrm{k}+\mathrm{g})_{1}(\mathrm{k}+\overline{\mathrm{g}})_{2}+ \\
& c_{66}(\overline{\mathbf{g}}-\mathbf{g})(\mathrm{k}+\mathrm{g})_{2}(\mathrm{k}+\overline{\mathrm{g}})_{1}, \\
& \mathbf{K}_{22}=c_{11}(\overline{\mathbf{g}}-\mathbf{g})(\mathrm{k}+\mathrm{g})_{2}(\mathrm{k}+\overline{\mathrm{g}})_{2}+ \\
& c_{66}(\overline{\mathbf{g}}-\mathbf{g})(\mathrm{k}+\mathrm{g})_{1}(\mathrm{k}+\overline{\mathrm{g}})_{1}, \\
& \mathbf{K}_{33}=c_{44}(\overline{\mathbf{g}}-\mathbf{g})(\mathrm{k}+\mathrm{g})_{1}(\mathrm{k}+\overline{\mathrm{g}})_{1}+ \\
& c_{44}(\overline{\mathbf{g}}-\mathbf{g})(\mathrm{k}+\mathrm{g})_{2}(\mathrm{k}+\overline{\mathrm{g}})_{2}, \\
& \mathbf{K}_{34}=e_{15}(\overline{\mathbf{g}}-\mathbf{g})(\mathrm{k}+\mathrm{g})_{1}(\mathrm{k}+\overline{\mathrm{g}})_{1}+ \\
& e_{15}(\overline{\mathbf{g}}-\mathbf{g})(\mathrm{k}+\mathrm{g})_{2}(\mathrm{k}+\overline{\mathrm{g}})_{2}, \\
& \mathbf{K}_{44}=-\epsilon_{11}(\overline{\mathbf{g}}-\mathbf{g})(\mathrm{k}+\mathrm{g})_{1}(\mathrm{k}+\overline{\mathrm{g}})_{1}- \\
& \epsilon_{11}(\overline{\mathbf{g}}-\mathbf{g})(\mathrm{k}+\mathrm{g})_{2}(\mathrm{k}+\overline{\mathrm{g}})_{2}, \\
& \mathbf{K}_{43}=\mathbf{K}{ }_{34} \cdot
\end{aligned}
$$

The matrix $\mathbf{M}$ in Eq. (13) is expressed by:

$$
\mathbf{M}=\left[\begin{array}{cccc}
\rho(\overline{\mathbf{g}}-\mathbf{g}) & \mathbf{0} & \mathbf{0} & \mathbf{0} \\
\mathbf{0} & \rho(\overline{\mathbf{g}}-\mathbf{g}) & \mathbf{0} & \mathbf{0} \\
\mathbf{0} & \mathbf{0} & \rho(\overline{\mathbf{g}}-\mathbf{g}) & \mathbf{0} \\
\mathbf{0} & \mathbf{0} & \mathbf{0} & \mathbf{0}
\end{array}\right]
$$

The vector $\mathbf{q}$ in Eq. (13) is given by:

$$
\mathbf{q}=\left\{\begin{array}{l}
u_{1 \mathbf{k}}(\mathbf{g}) \\
u_{2 \mathbf{k}}(\mathbf{g}) \\
u_{3 \mathbf{k}}(\mathbf{g}) \\
\phi_{\mathbf{k}}(\mathbf{g})
\end{array}\right] .
$$

Equation (13) is an infinite system of equations, thus the Fourier series needs to be truncated. We choose $m, \bar{m}, n, \bar{n}$ $=[-M, \ldots, M]$ and the total number of plane waves is $(2 M$ $+1)^{2}$. Equation (13) represents a generalized eigenvalue problem of $\omega^{2}(\mathbf{k})$ and should be solved for each $\mathbf{k}$ into the FIBZ for square, rectangular, triangular, honeycomb and Kagomé lattices (Figure 1(f-h)).

The Fourier coefficients are defined as:

$$
P(\overline{\mathbf{g}})=\left\{\begin{array}{l}
f P_{A}+(1-f) P_{B} \text { for } \overline{\mathbf{g}}=\mathbf{0} \\
\left(P_{A}-P_{B}\right) F(\overline{\mathbf{g}}) \text { for } \overline{\mathbf{g}} \neq \mathbf{0}
\end{array},\right.
$$

where the indexes $A$ and B in Eq. (25) are related to inclusion $\left(\mathrm{BaTiO}_{3}\right)$ and polymeric matrix, respectively, $F(\overline{\mathbf{g}})$ is the structure function and $f$ is the filling fraction of each type of inclusion, considering circular section of radius $\tilde{r}$, square section of width $2 l$, rotated square section of width $2 l$ with a $45^{\circ}$ angle of rotation with respect to $x$ and $y$ axes and hollow circular section with external radius $\tilde{R}$ and internal radius $\tilde{r}$, $\tilde{R}>\tilde{r}$. The hollow cylinder inclusion has an internal radius $\tilde{\mathrm{r}}$ of the same polymer at matrix and a thickness $\tilde{R}-\tilde{r}$ of $\mathrm{BaTiO}_{3}$, similar to Anjos \& Arantes ${ }^{10}$.

The filling fraction is defined as $f=S_{A} / S_{C}$ for square, rectangular and triangular lattices, whereas for honeycomb and Kagomé lattices it is defined as $f=2 S_{A} / S_{C}$ and $f=3 S_{A} / S_{C}$, respectively, where $S_{A}$ is the cross section area of the $\mathrm{BaTiO}_{3}$ inclusions and $S_{C}=\left\|\mathbf{a}_{1} \times \mathbf{a}_{2}\right\|$ is the cross section area of the unit cell. Note that differently from other lattices honeycomb and Kagomé lattices have two and three inclusions per unit cell, respectively.

The filling fractions for square, rectangular, triangular, honeycomb and Kagomé lattices are:

$$
f=\left\{\begin{array}{l}
\pi \tilde{r}^{2} / a^{2} \text { for circular sec tion } \\
4 l^{2} / a^{2} \text { for square sec tion } \\
4 l^{2} / a^{2} \text { for rotated square sec tion } \\
\pi\left(\tilde{R}^{2}-\tilde{r}^{2}\right) / a^{2} \text { for hollow sec tion }
\end{array}\right.
$$


$f=\left\{\begin{array}{l}\pi \tilde{r}^{2} /\left(a_{1} a_{2}\right) \text { for circular sec tion } \\ 4 l^{2} /\left(a_{1} a_{2}\right) \text { for square sec tion } \\ 4 l^{2} /\left(a_{1} a_{2}\right) \text { for rotated square sec tion } \\ \pi\left(\tilde{R}^{2}-\tilde{r}^{2}\right) /\left(a_{1} a_{2}\right) \text { for hollow sec tion }\end{array}\right.$,

$f=\left\{\begin{array}{l}2 \pi \tilde{r}^{2} / \sqrt{3} a^{2} \text { for circular sec tion } \\ 8 l^{2} / \sqrt{3} a^{2} \text { for square sec tion } \\ 8 l^{2} / \sqrt{3} a^{2} \text { for rotated square sec tion } \\ 2 \pi\left(\tilde{R}^{2}-\tilde{r}^{2}\right) / \sqrt{3} a^{2} \text { for hollow sec tion }\end{array}\right.$,

$f=\left\{\begin{array}{l}4 \pi \tilde{r}^{2} / 3 \sqrt{3} a^{2} \text { for circular sec tion } \\ 16 l^{2} / 3 \sqrt{3} a^{2} \text { for square sec tion } \\ 16 l^{2} / 3 \sqrt{3} a^{2} \text { for rotated square sec tion } \\ 4 \pi\left(\tilde{R}^{2}-\tilde{r}^{2}\right) / 3 \sqrt{3} a^{2} \text { for hollow sec tion }\end{array}\right.$

$f=\left\{\begin{array}{l}\pi \sqrt{3} \tilde{r}^{2} / 2 a^{2} \text { for circular sec tion } \\ 2 \sqrt{3} l^{2} / a^{2} \text { for square sec tion } \\ 2 \sqrt{3} l^{2} / a^{2} \text { for rotated square sec tion } \\ \pi \sqrt{3}\left(\tilde{R}^{2}-\tilde{r}^{2}\right) / 2 a^{2} \text { for hollow section }\end{array}\right.$,

respectively.

The structure function for square, rectangular and triangular lattices $F(\overline{\mathbf{g}})$ is given by:

$$
F(\overline{\mathbf{g}})=\frac{1}{S_{c}} \iint e^{-j \overline{\mathbf{g}} \cdot \mathbf{r}} d^{2} r .
$$

The integral in Eq. (31) is performed over the unit cell cross section. The structure functions for square, rectangular and triangular lattices are calculated by:

$$
F(\overline{\mathbf{g}})=\left\{\begin{array}{l}
2 f J_{1}(\bar{g} \tilde{r}) / \bar{g} \tilde{r} \text { for circular sec tion } \\
f\left[\sin \left(\bar{g}_{1} l\right) / \bar{g}_{1} l\right]\left[\sin \left(\bar{g}_{2} l\right) / \bar{g}_{2} l\right] \text { for square sec tion } \\
f\left\{\frac{\left.\left.\sin \left[(l / \sqrt{2})\left(\bar{g}_{1}+\bar{g}_{2}\right)\right]\right]\right\}\left\{\sin \left[(l / \sqrt{2})\left(-\bar{g}_{1}+\bar{g}_{2}\right)\right]\right.}{\left[(l / \sqrt{2})\left(\bar{g}_{1}+\bar{g}_{2}\right)\right]}\right\}\left\{\frac{\left[(l / \sqrt{2})\left(-\bar{g}_{1}+\bar{g}_{2}\right)\right]}{\text { for square rotated sec tion }}\right. \\
2 f\left[J_{1}(\bar{g} \tilde{R})-(\tilde{r} / \tilde{R}) J_{1}(\bar{g} \tilde{R})\right] /(\bar{g} \tilde{R}) \text { for hollow sec tion }
\end{array}\right.
$$

where $\bar{g}=\|\overline{\mathbf{g}}\|$ and $\bar{g}_{1,2}=\left\|\overline{\mathbf{g}}_{1,2}\right\|$.

The structure function for honeycomb lattice $F_{H}(\overline{\mathbf{g}})$ is defined $a^{84}$ :

$$
\begin{aligned}
& F_{H}(\overline{\mathbf{g}})=\cos \left(\overline{\mathbf{g}} \cdot \overline{\mathbf{u}}_{1}\right) \frac{1}{S_{c}} \iint e^{-j \overline{\mathbf{g}} \cdot \mathbf{r}} d^{2} r= \\
& \cos \left(\overline{\mathbf{g}} \cdot \overline{\mathbf{u}}_{1}\right) F(\overline{\mathbf{g}}),
\end{aligned}
$$

where $\overline{\mathbf{u}}_{1}=-\overline{\mathbf{u}}_{2}=a(0,1 / 2)$ are vectors that define central position of the two $\mathrm{BaTiO}_{3}$ inclusions within the honeycomb unit cell. We choose these vectors similar to Gao et al. ${ }^{55}$. Thus, the structure functions of $\mathrm{BaTiO}_{3}$ inclusions for honeycomb lattice are the same of Eq. (31) multiplied by $\cos \left(\overline{\mathbf{g}} \cdot \overline{\mathbf{u}}_{1}\right)$, considering $f$ from Eq. (29).
Table 1. Physical parameters of $\mathrm{BaTiO}_{3}$ inclusion (A) and polymeric matrix (B).

\begin{tabular}{lc}
\hline Geometry/Property & Value \\
\hline Lattice parameter $(a)$ & $30 \times 10^{-9} \mathrm{~m}$ \\
Lattice parameter $\left(a_{1}\right)$ & $30 \times 10^{-9} \mathrm{~m}$ \\
Lattice parameter $\left(a_{2}\right)$ & $25 \times 10^{-9} \mathrm{~m}$ \\
Filling fraction $(f)$ & 0.335 \\
Mass density $\left(\rho_{A}, \rho_{B}\right)$ & $5730 \mathrm{~kg} / \mathrm{m}^{3}, 1150 \mathrm{~kg} / \mathrm{m}^{3}$ \\
Elastic constant $\left(c_{11 A}, c_{11 B}\right)$ & $166 \times 10^{9} \mathrm{~N} / \mathrm{m}^{2}, 7.8 \times 10^{9} \mathrm{~N} / \mathrm{m}^{2}$ \\
Elastic constant $\left(c_{12 A}, c_{12 B}\right)$ & $77 \times 10^{9} \mathrm{~N} / \mathrm{m}^{2}, 4.7 \times 10^{9} \mathrm{~N} / \mathrm{m}^{2}$ \\
Elastic constant $\left(c_{44 A}, c_{44 B}\right)$ & $43 \times 10^{9} \mathrm{~N} / \mathrm{m}^{2}, 1.6 \times 10^{9} \mathrm{~N} / \mathrm{m}^{2}$ \\
Elastic constant $\left(c_{66 A}, c_{66 B}\right)$ & $44.5 \times 10^{9} \mathrm{~N} / \mathrm{m}^{2}, 1.55 \times 10^{9} \mathrm{~N} / \mathrm{m}^{2}$ \\
Piezoelectric & $11.6 \mathrm{C}^{2} \mathrm{~m}^{2}, 0 \mathrm{C} / \mathrm{m}^{2}$ \\
coefficient $\left(e_{15 A}, e_{15 B}\right)$ & $11.2 \times 10^{-9} \mathrm{C}^{2} / \mathrm{Nm}{ }^{2}, 0.0398 \times$ \\
Dielectric coefficient & $10^{-9} \mathrm{C}^{2} / \mathrm{Nm}^{2}$ \\
$\left(\epsilon_{11 A}, \epsilon_{11 B}\right)$ &
\end{tabular}

Note that $a$ is the lattice parameter for square, triangular, honeycomb and Kagomé lattices, and $a_{1}, a_{2}$ are the lattice parameters for rectangular lattice.

The structure function for Kagomé lattice $F_{K g}(\overline{\mathbf{g}})$ is defined as:

$$
\begin{aligned}
& F_{K g}(\overline{\mathbf{g}})=\frac{1}{3} \sum_{i=1}^{3} e^{j(\overline{\mathbf{g}} \cdot \overline{\mathbf{u}})} \frac{1}{S_{c}} \iint e^{-j \overline{\mathbf{g}} \cdot \mathbf{r}} d^{2} r= \\
& \frac{1}{3} \sum_{i=1}^{3} e^{-j(\overline{\mathbf{g}} \cdot \overline{\mathbf{u}})} F(\overline{\mathbf{g}})
\end{aligned}
$$

where $\overline{\mathbf{u}}_{1}=-\frac{a}{2} \mathbf{e}_{1}-\frac{\sqrt{3 a}}{6} \mathbf{e}_{2}, \overline{\mathbf{u}}_{2}=\frac{a}{2} \mathbf{e}_{1}-\frac{\sqrt{3 a}}{6} \mathbf{e}_{2}, \overline{\mathbf{u}}_{3}=\frac{\sqrt{3 a}}{3} \mathbf{e}_{1}$ are vectors that define the three $\mathrm{BaTiO}_{3}$ inclusion positions within the Kagomé unit cell. It is possible to split Eq. (34) in its real and imaginary parts:

$$
\begin{aligned}
& F_{k g}(\overline{\mathbf{g}})=\operatorname{Re}\left[F_{k g}(\overline{\mathbf{g}})\right]+j \operatorname{Im}\left[F_{k g}(\overline{\mathbf{g}})\right] \\
& \operatorname{Re}\left[F_{k g}(\overline{\mathbf{g}})\right]=\left[2 \cos \left(\overline{\mathrm{g}}_{1} \bar{u}_{11}\right) \cos \left(\overline{\mathrm{g}}_{2} \bar{u}_{12}\right)+\cos \left(2 \overline{\mathrm{g}}_{2} \bar{u}_{12}\right)\right] F(\overline{\mathbf{g}}),
\end{aligned}
$$

$\operatorname{Im}\left[F_{k g}(\overline{\mathrm{g}})\right]=\left[-2 \cos \left(\overline{\mathrm{g}}_{1} \bar{u}_{1_{1}}\right) \sin \left(\overline{\mathrm{g}}_{2} \bar{u}_{1_{2}}\right)+\sin \left(2 \overline{\mathrm{g}}_{1} \bar{u}_{1_{2}}\right)\right] F(\overline{\mathbf{g}})$,

where $\bar{u}_{j 1,2}=\left\|\overline{\mathbf{u}}_{\mathrm{j} 1,2}\right\|$.

\section{Results and Discussion}

The physical parameters of $\mathrm{BaTiO}_{3}$ inclusion (A) and polymeric matrix (B) are listed in Table 1 . We calculate the elastic band structure considering initially a fixed filling fraction, 0.335 , for the four inclusion cross section geometries considered - circular, hollow circular, square and rotated square with a $45^{\circ}$ angle of rotation with respect to the $x, y$ axes, in a square, rectangular, triangular, honeycomb and 

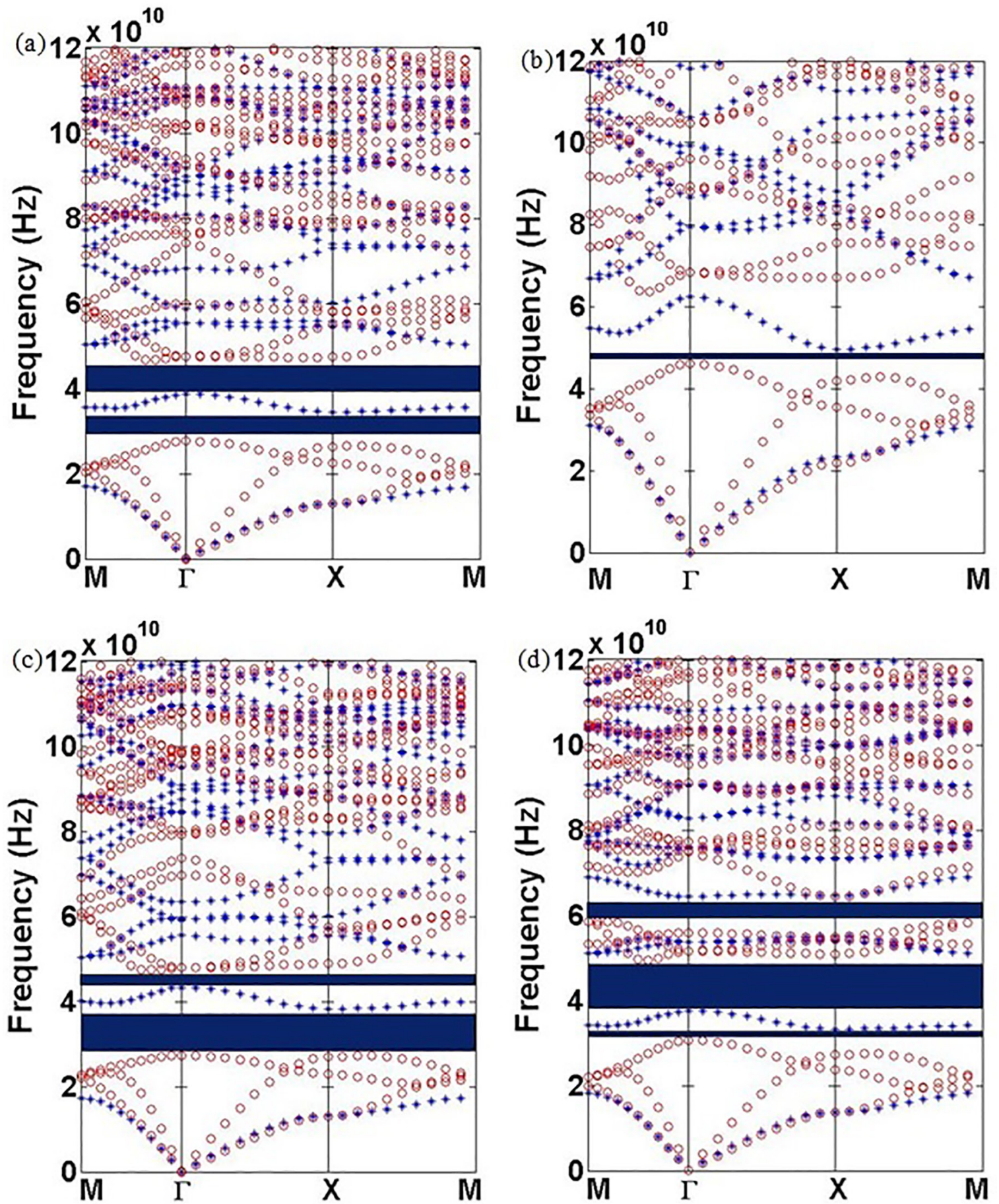

Figure 2. Elastic band structures of $\mathrm{XY}$ (red) and $\mathrm{Z}$ (blue) modes of $\mathrm{BaTiO}_{3}$ inclusions in a polymeric matrix for square lattice. The following types of inclusions are considered - circular (a), hollow circular (b), square (c) and rotated square with a $45^{\circ}$ angle of rotation with respect to the $x, y$ axes. 

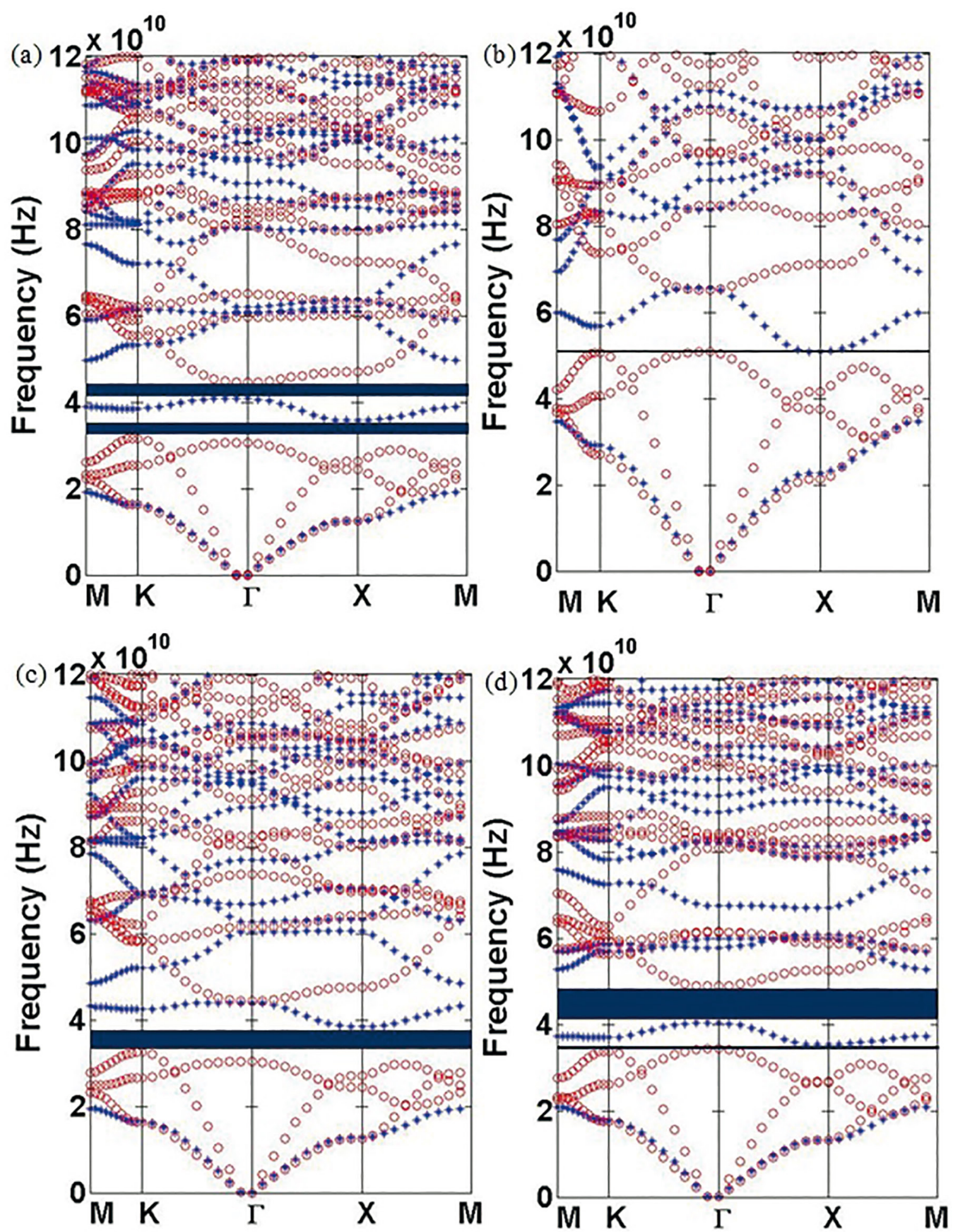

Figure 3. Elastic band structures of $\mathrm{XY}$ (red) and $\mathrm{Z}$ (blue) modes of $\mathrm{BaTiO}_{3}$ inclusions in a polymeric matrix for rectangular lattice. The following types of inclusions are considered - circular (a), hollow circular (b), square (c) and rotated square with a $45^{\circ}$ angle of rotation with respect to the $x, y$ axes. 
Kagomé lattices. In the course of numerical calculations, we consider 441 plane waves for Fourier series expansion (i.e. $M$ $=10$ ), which results in a good convergence. Band structure plots are limited until a maximum frequency of $0.12 \mathrm{THz}$.

Figure 2 (a-d) shows the band structures of the nanopiezoelectric $\mathrm{PC}$ for square lattice, considering the four types of inclusions and XY (red) and Z (blue) modes. We plot the band structures in the principal symmetry directions of FIBZ (see Figure $1(\mathrm{f}-\mathrm{h})$ ). Plots are given in terms of frequency in $\mathrm{Hz}$ versus the reduced Bloch wave vector, $\overline{\mathbf{k}}=\mathbf{k} a / 2 \pi$. The reduced Bloch wave vector for rectangular lattice is calculated taking into account $a=\sqrt{a_{1}^{2}+a_{2}^{2}}$. In Figure 2 (a), two complete band gaps are obtained for circular inclusion.

From now on the relation between parameters $\tilde{R}$ and $\tilde{r}$ for hollow circular inclusion is $\tilde{r}=0.2 \tilde{R}$ and we do not investigate the influence of $\mathrm{BaTiO}_{3}$ thickness, i.e. $\tilde{R}-\tilde{r}$, in the band structure. The thickness influence of a carbon microstructure inclusion for square lattice was investigated by Anjos \& Arantes ${ }^{10}$. Figure 2 (b) presents one complete band gap for hollow circular inclusion and we can observe that first branches occur in higher frequencies compared to the other inclusions.

Figure 2 (c) shows two complete band gaps for square inclusion and its first complete band gap is broader than the complete band gaps for circular and hollow circular inclusions. When these square inclusions are rotated $45^{\circ}$ with respect to $x$ and $y$ axes (Figure $2(\mathrm{~d})$ ) three complete gaps are opened up. The broadest complete band gap for square lattice is for rotated square inclusion with a bandwidth of 11.97 $\mathrm{GHz}$ (43.5 GHz is the center frequency).

Figure 3 (a-d) shows the band structures for rectangular lattice. For circular and hollow circular inclusions, the band gap behavior of rectangular lattice is similar to square lattice, however they are narrower than the complete band gaps in a square lattice. For square and rotated square inclusions, only one and two complete band gaps are opened up, respectively. Similar to square lattice, the broadest complete band gap for rectangular lattice is opened up for rotated square inclusion with a bandwidth of $8.51 \mathrm{GHz}(44.59 \mathrm{GHz}$ is the center frequency).

Figure 4 (a-d) illustrates the band structures for triangular lattice. For circular, square and rotated square inclusions, a wide complete band gap is created. The broadest complete band gap for triangular lattice is for circular inclusion with a bandwidth of $12.98 \mathrm{GHz}$ ( $36.31 \mathrm{GHz}$ is the center frequency). A narrow complete band gap is also opened up for circular inclusion in high bands. For hollow inclusion, one complete band gap is created between $58.69 \mathrm{GHz}-49.94 \mathrm{GHz}$. Comparing the band structures of triangular lattice to square and rectangular lattices, we observe that triangular lattice with circular inclusion presents the broadest complete band gap.
In Figures 5 (a-d) and 6 (a-d), honeycomb and Kagomé lattices, respectively, the band structures of square and rotated square inclusions present similar band gap behavior. The band structure of circular inclusion in honeycomb and Kagomé lattices presents seven and five complete band gaps, respectively. For Kagomé lattice, all the complete band gaps are narrower than the broadest complete band gap from square, rectangular, triangular and honeycomb lattices. The broadest complete band gap for honeycomb lattice is for circular inclusion with a bandwidth of $9.77 \mathrm{GHz}$ ( $31.19 \mathrm{GHz}$ is the center frequency). For hollow inclusion in honeycomb and Kagomé lattices, we can observe two and one complete band gaps, respectively, and the first bands appear in higher frequencies compared to the other inclusions, similar as observed to other lattices - square, rectangular and triangular.

Comparing the band structures for honeycomb and Kagomé lattices, we observe that honeycomb lattice presents the best performance (more complete band gaps and broader complete band gaps) for all inclusions.

In Figures 7-11, we show the comparison between $\mathrm{Z}$ modes of the nano-piezoelectric PC with (blue asterisks) and without $\left(e_{15 A}=0\right)$ (black circles) piezoelectricity for square, rectangular, triangular, honeycomb and Kagomé lattices, respectively. The influence of piezoelectricity is significant on the band gaps for all lattices and inclusions. This influence is more evident in high frequencies ${ }^{60}$ for circular, square and rotated square inclusions. However, for hollow circular inclusion in all lattices (Figures 7-11 (b)), the piezoelectricity is also significant in lower frequencies.

We illustrate in Figures 12-16 the complete band gap widths between $\mathrm{XY}$ and $\mathrm{Z}$ modes as a function of filling fraction for all inclusions and lattices. Piezoelectricity is included in this analysis.

In a square lattice (Figure 12), circular and square inclusions provide the best behavior with high complete band gap widths in a broad range of filling fraction. Considering the first band gap, square inclusion shows the highest one in a broad range of filling fraction. Furthermore, square inclusion also presents four complete band gaps. Hollow circular inclusion presents only one complete band gap, whereas rotated square inclusion does not present better performance than square inclusion.

For rectangular lattice, Figure 13, hollow circular inclusion opens up just one narrow complete band gap, whereas rotated square inclusion creates three complete band gaps, however, only the second one appear in a broad range of filling fraction. Similar as square lattice (Figure 12), circular and square inclusions present the broadest complete band gaps for rectangular lattice. Complete band gap widths in rectangular lattice are lower than in square lattice for all inclusions. 

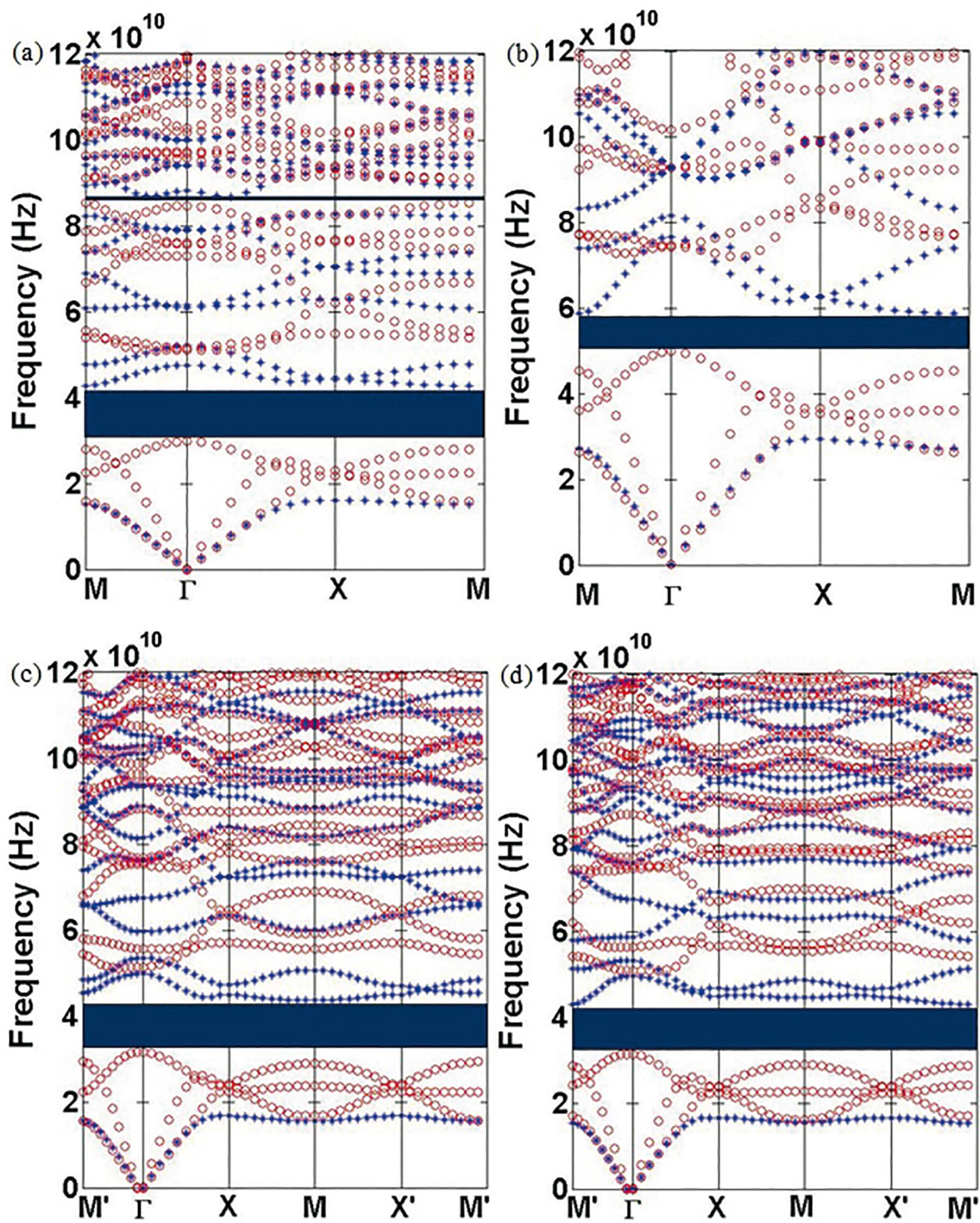

Figure 4. Elastic band structures of $\mathrm{XY}$ (red) and $\mathrm{Z}$ (blue) modes of $\mathrm{BaTiO}_{3}$ inclusions in a polymeric matrix for triangular lattice. The following types of inclusions are considered - circular (a), hollow circular (b), square (c) and rotated square with a $45^{\circ}$ angle of rotation with respect to the $x, y$ axes. 

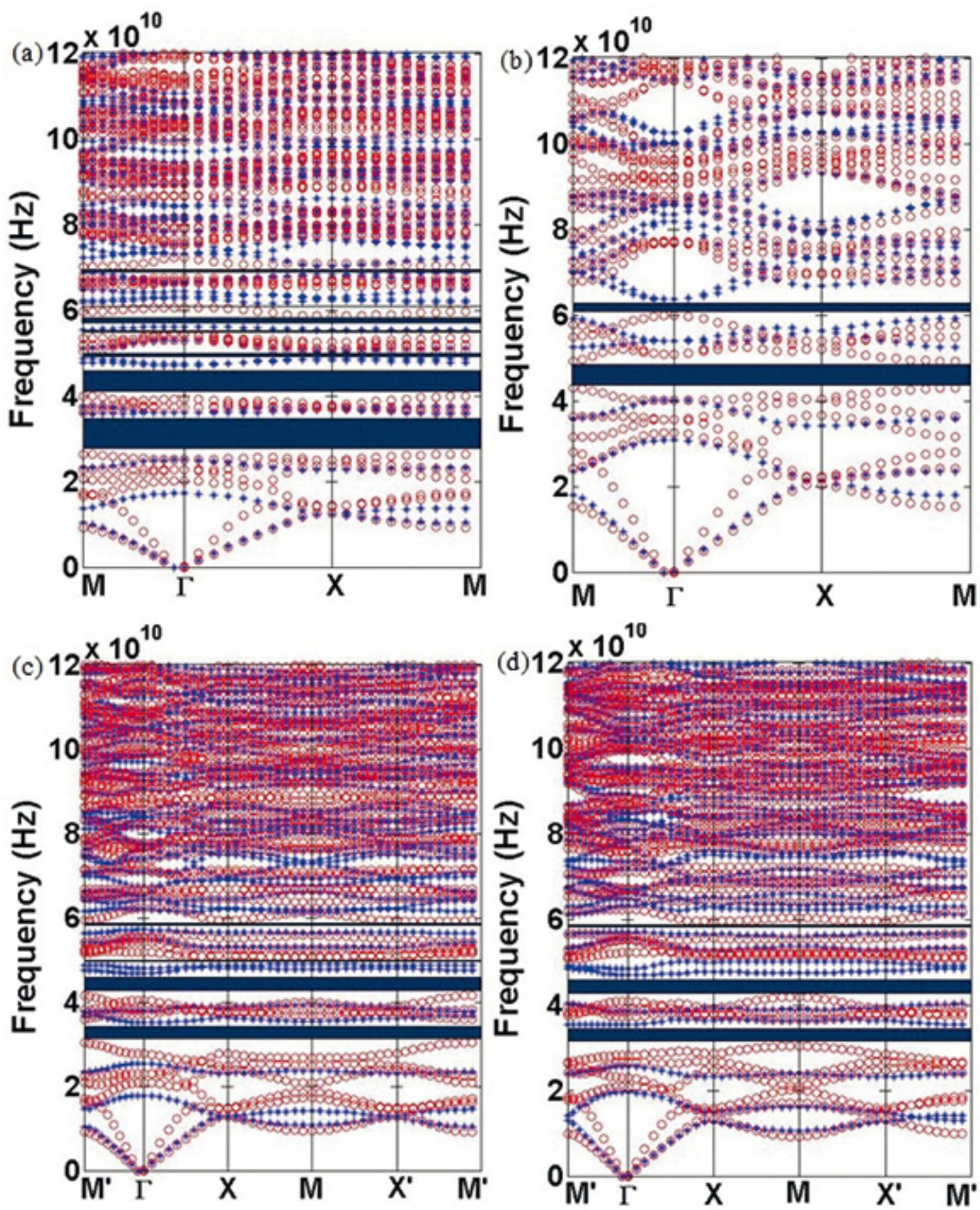

Figure 5. Elastic band structures of $\mathrm{XY}$ (red) and $\mathrm{Z}$ (blue) modes of $\mathrm{BaTiO}_{3}$ inclusions in a polymeric matrix for honeycomb lattice. The following types of inclusions are considered - circular (a), hollow circular (b), square (c) and rotated square with a $45^{\circ}$ angle of rotation with respect to the $x, y$ axes. 

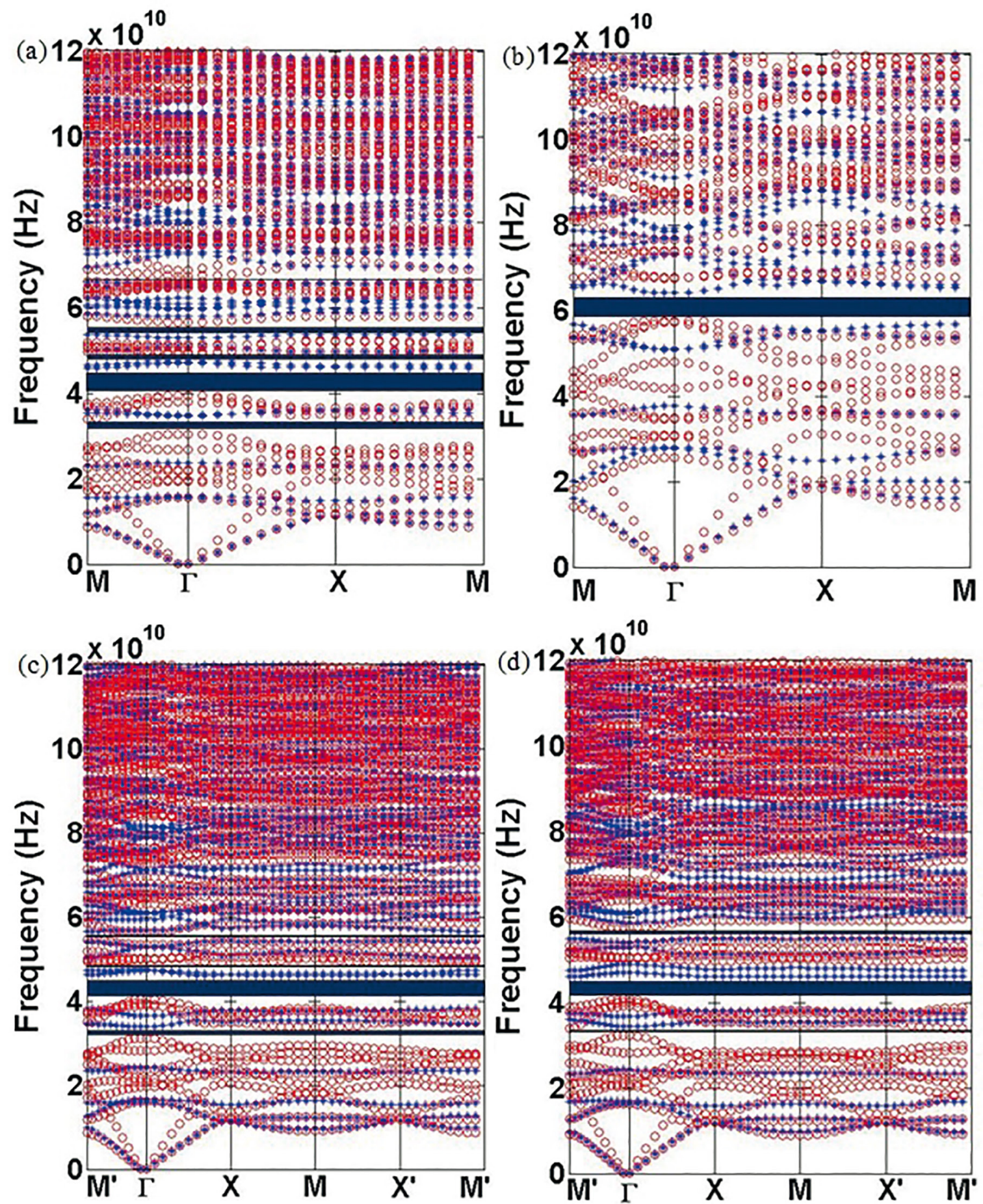

Figure 6. Elastic band structures of $\mathrm{XY}$ (red) and $\mathrm{Z}$ (blue) modes of $\mathrm{BaTiO}_{3}$ inclusions in a polymeric matrix for Kagomé lattice. The following types of inclusions are considered - circular (a), hollow circular (b), square (c) and rotated square with a $45^{\circ}$ angle of rotation with respect to the $x, y$ axes. 

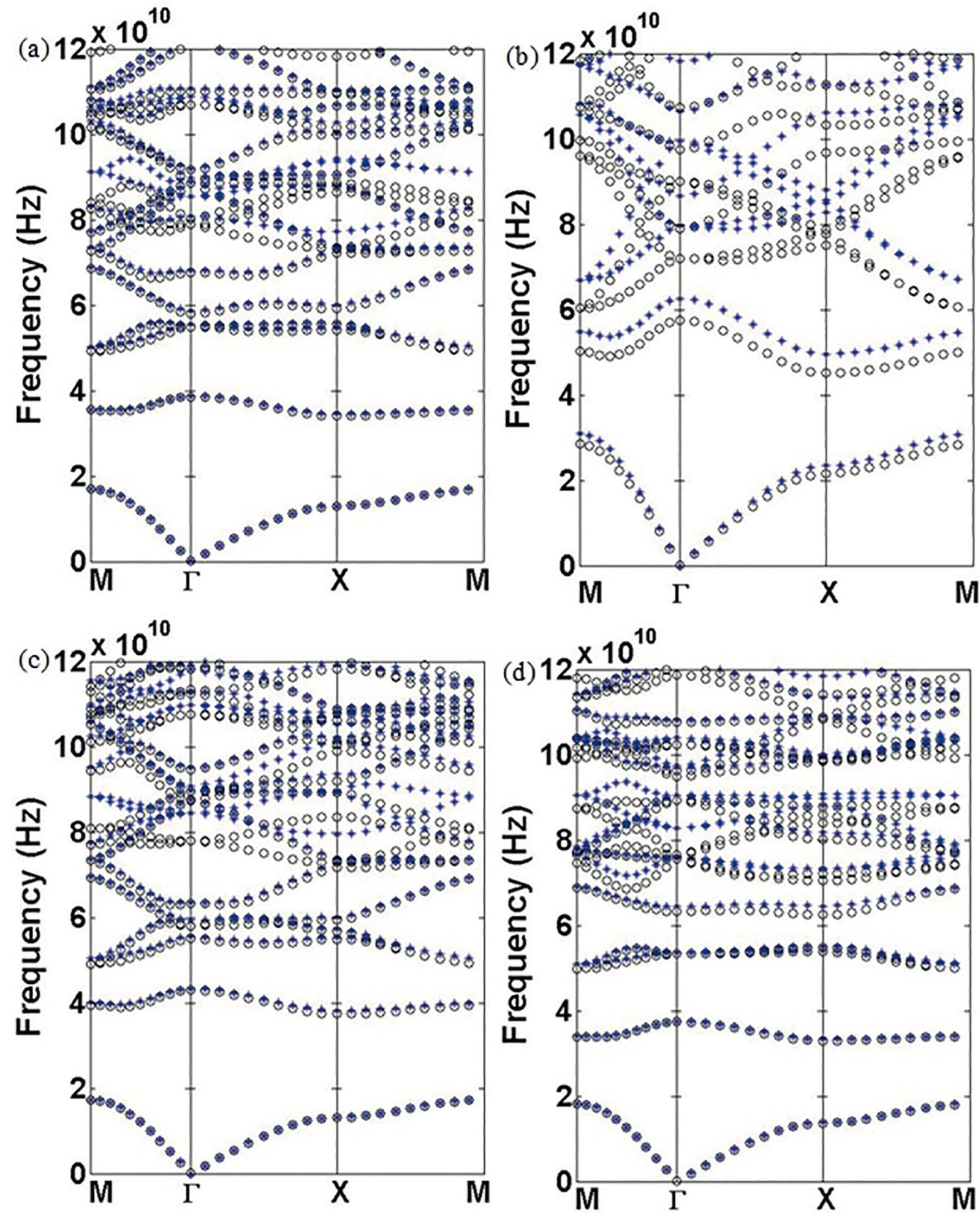

Figure 7. Elastic band structures of $\mathrm{BaTiO}_{3}$ inclusions in a polymeric matrix considering $\mathrm{Z}$ mode with (blue asterisks) and without (black circles) piezoelectricity for square lattice. The following types of inclusions are considered - circular (a), hollow circular (b), square (c) and rotated square with a $45^{\circ}$ angle of rotation with respect to the $x, y$ axes. 

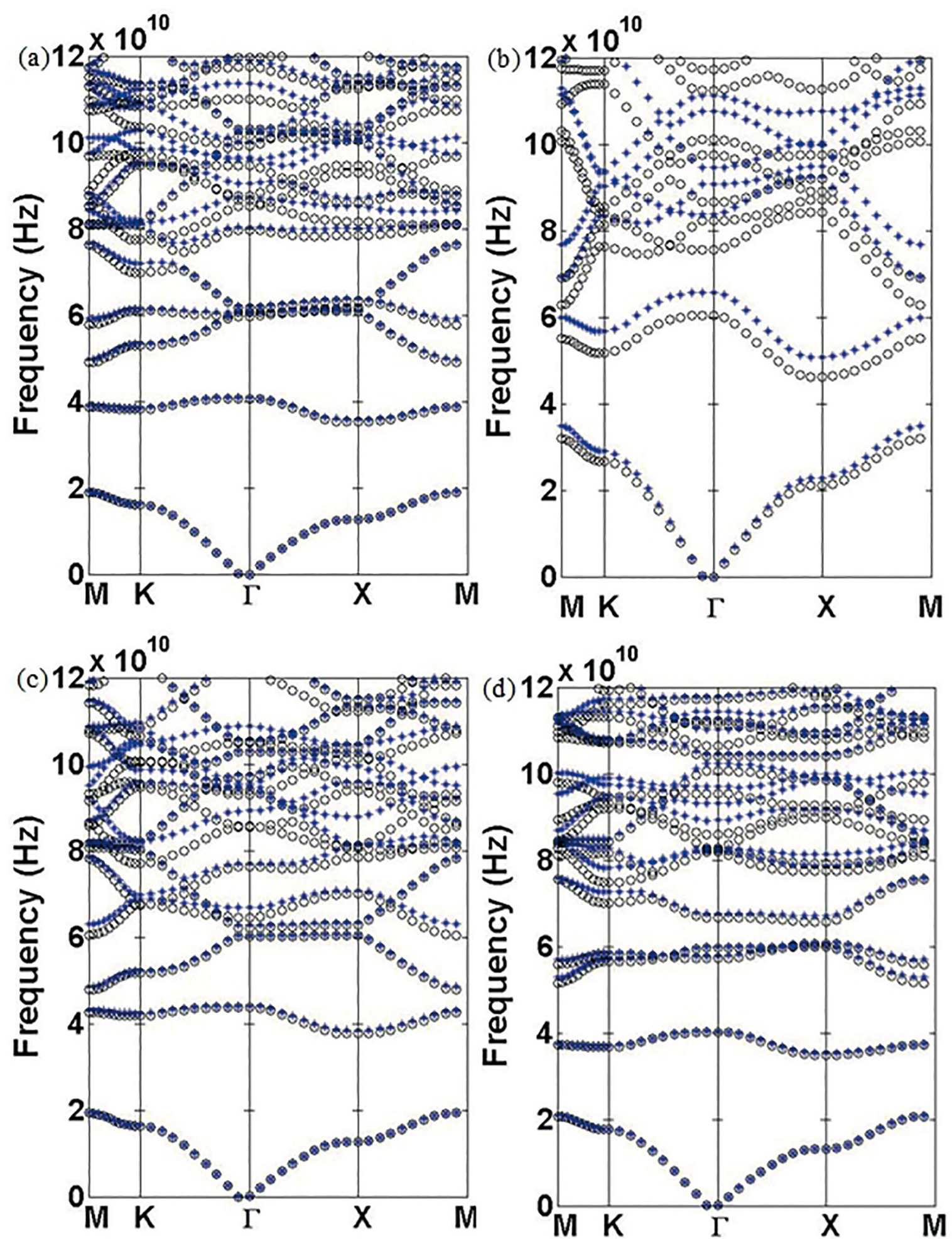

Figure 8. Elastic band structures of $\mathrm{BaTiO}_{3}$ inclusions in a polymeric matrix considering $\mathrm{Z}$ mode with (blue asterisks) and without (black circles) piezoelectricity for rectangular lattice. The following types of inclusions are considered - circular (a), hollow circular (b), square (c) and rotated square with a $45^{\circ}$ angle of rotation with respect to the $x, y$ axes. 

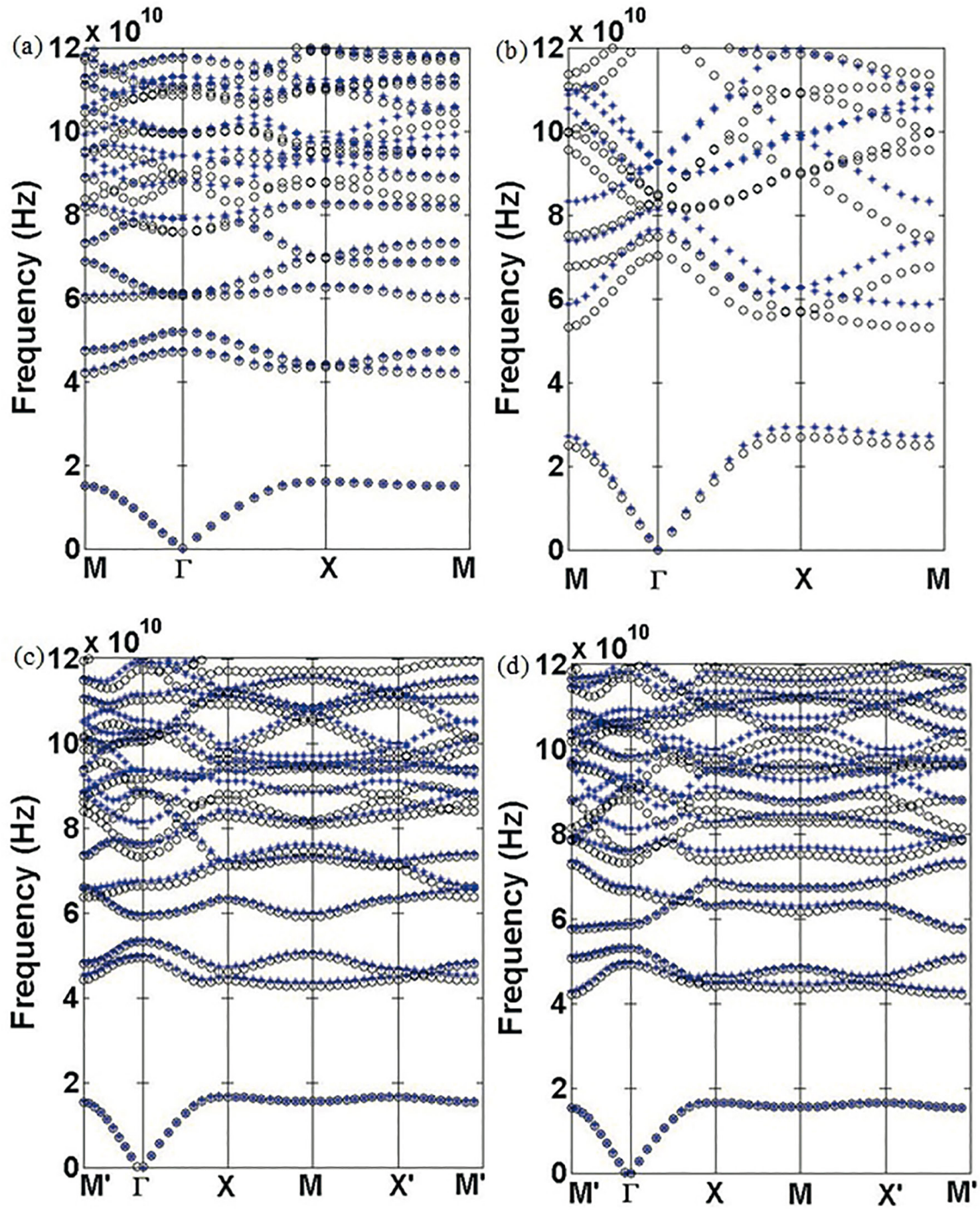

Figure 9. Elastic band structures of $\mathrm{BaTiO}_{3}$ inclusions in a polymeric matrix considering $\mathrm{Z}$ mode with (blue asterisks) and without (black circles) piezoelectricity for triangular lattice. The following types of inclusions are considered - circular (a), hollow circular (b), square (c) and rotated square with a $45^{\circ}$ angle of rotation with respect to the $x, y$ axes. 

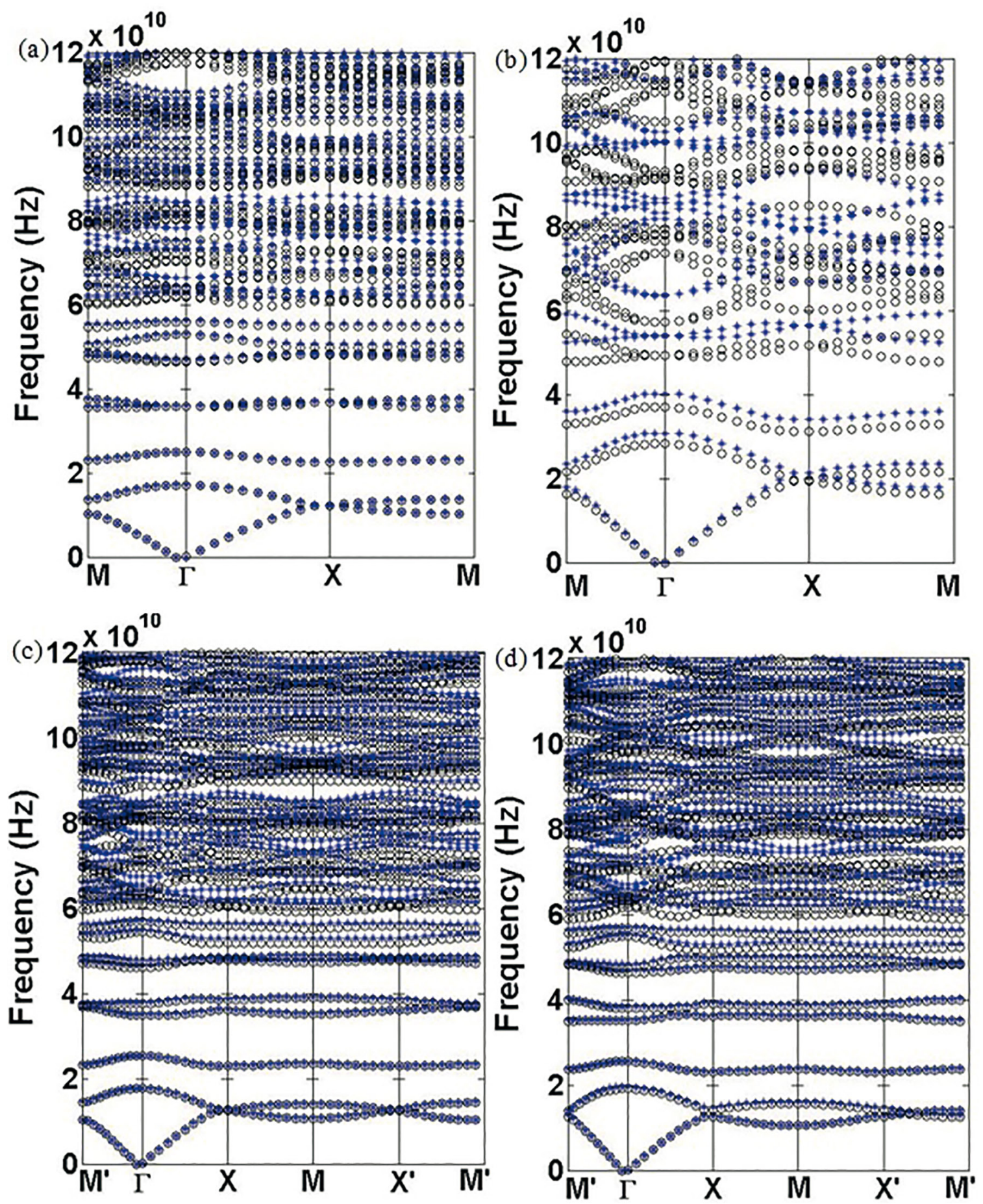

Figure 10. Elastic band structures of $\mathrm{BaTiO}_{3}$ inclusions in a polymeric matrix considering $\mathrm{Z}$ mode with (blue asterisks) and without (black circles) piezoelectricity for honeycomb lattice. The following types of inclusions are considered - circular (a), hollow circular (b), square (c) and rotated square with a $45^{\circ}$ angle of rotation with respect to the $x, y$ axes. 

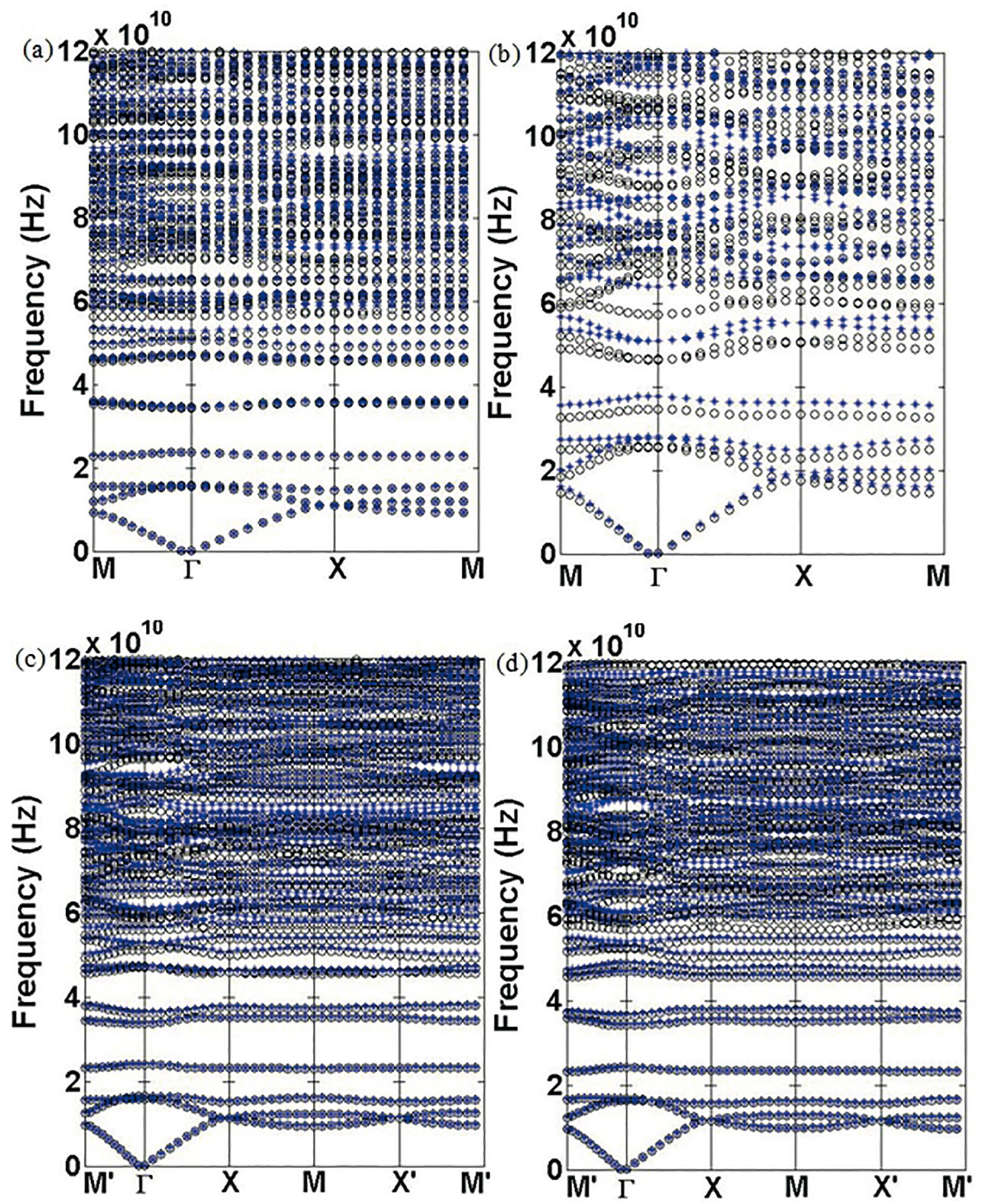

Figure 11. Elastic band structures of $\mathrm{BaTiO}_{3}$ inclusions in a polymeric matrix considering $\mathrm{Z}$ mode with (blue asterisks) and without (black circles) piezoelectricity for Kagomé lattice. The following types of inclusions are considered - circular (a), hollow circular (b), square (c) and rotated square with a $45^{\circ}$ angle of rotation with respect to the $x, y$ axes. 

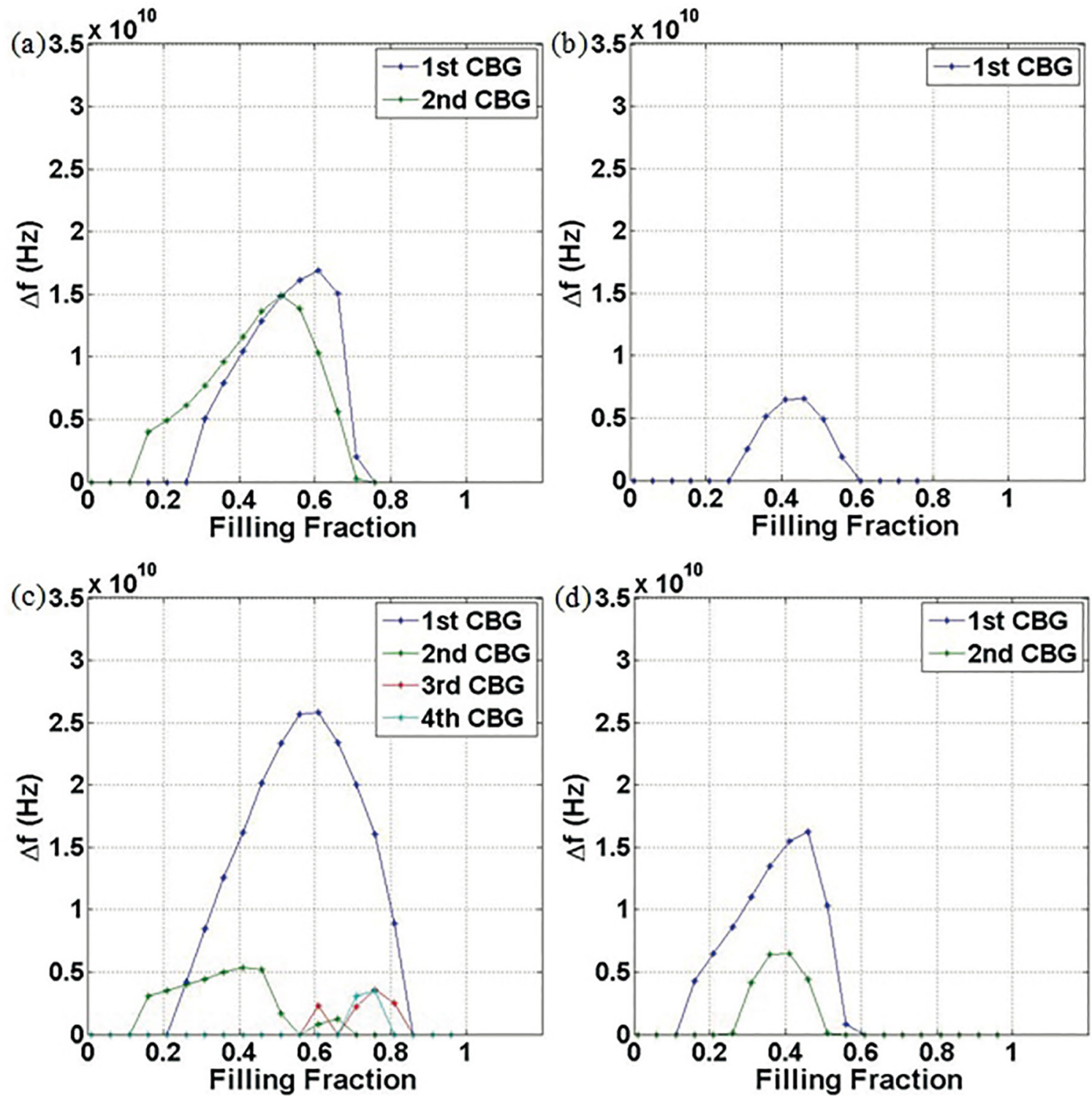

Figure 12. Elastic complete band gap widths between $\mathrm{XY}$ and $\mathrm{Z}$ modes of $\mathrm{BaTiO}_{3}$ inclusions in a polymeric matrix as a function of filling fraction for square lattice. The following types of inclusions are considered - circular (a), hollow circular (b), square (c) and rotated square with a $45^{\circ}$ angle of rotation with respect to the $x, y$ axes.

Figure 14 shows complete band gap widths for triangular lattice. Circular inclusion presents the best performance. Comparing triangular lattice (Figure 14) to square lattice (Figure 12), triangular lattice presents the best performance for circular (first complete band gap), hollow circular and rotated square inclusions. However, for square inclusion, square lattice shows better performance than triangular lattice. There are two important variables to conclude which is the best band gap performance, that is to say the band gap width (the higher, the better) and the filling fraction range in which the band gaps are opened (the broader, the better).
Comparing honeycomb (Figure 15) and Kagomé (Figure 16) lattices, the best performance is found for honeycomb lattice considering circular, hollow circular and rotated square inclusions. However, the difference among these lattices is subtle. The highest band gap width for honeycomb and Kagomé lattices is for circular inclusion. Comparing honeycomb and Kagomé lattices to the other lattices, we can observe that for all inclusions, the best behavior is found for square (Figure 12) and triangular (Figure 14) lattices. The worst performance is found for rectangular lattice with hollow circular inclusion (Figure 13 (b)). 

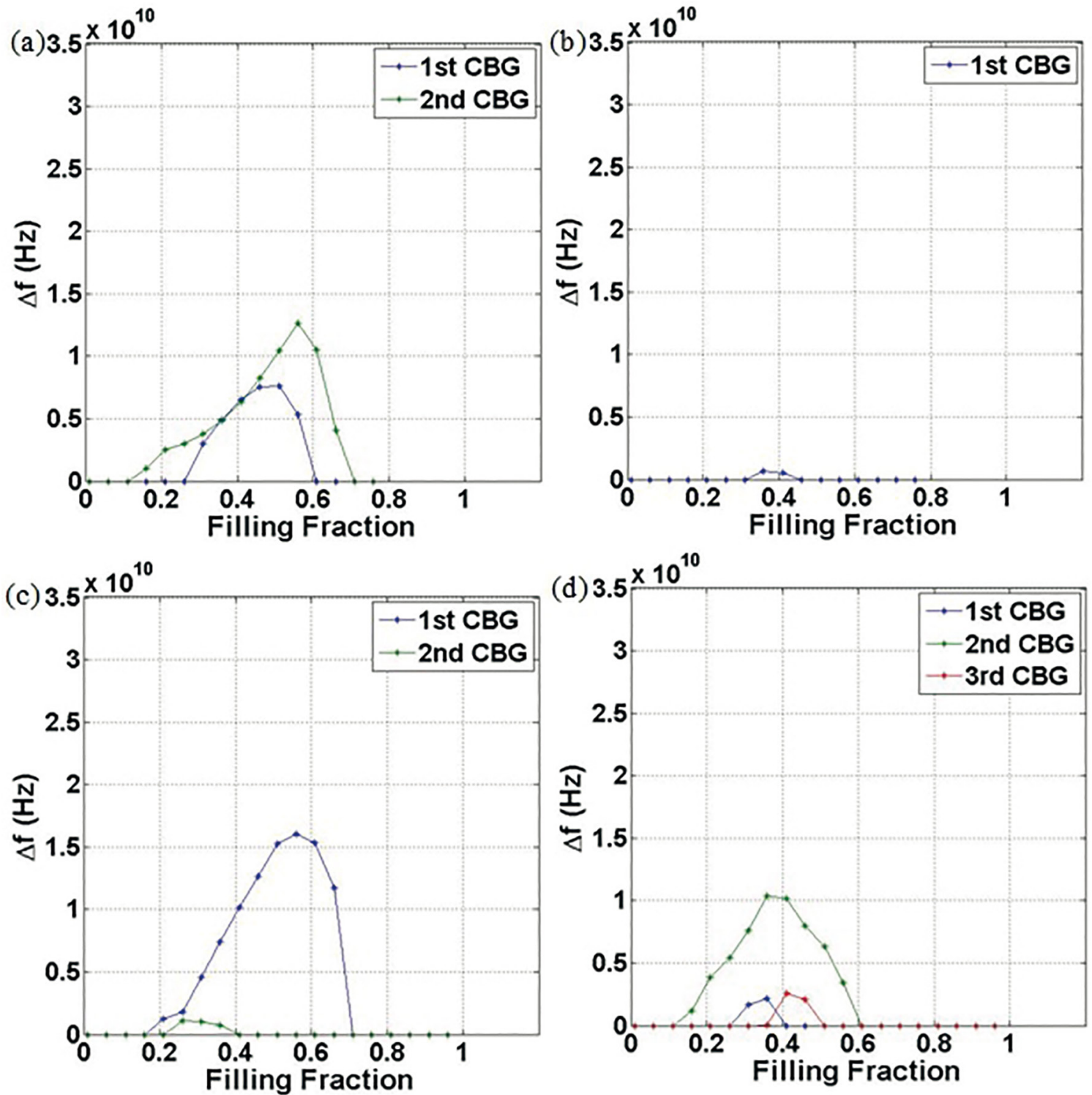

Figure 13. Elastic complete band gap widths between $\mathrm{XY}$ and $\mathrm{Z}$ modes of $\mathrm{BaTiO}_{3}$ inclusions in a polymeric matrix as a function of filling fraction for rectangular lattice. The following types of inclusions are considered - circular (a), hollow circular (b), square (c) and rotated square with a $45^{\circ}$ angle of rotation with respect to the $x, y$ axes. 

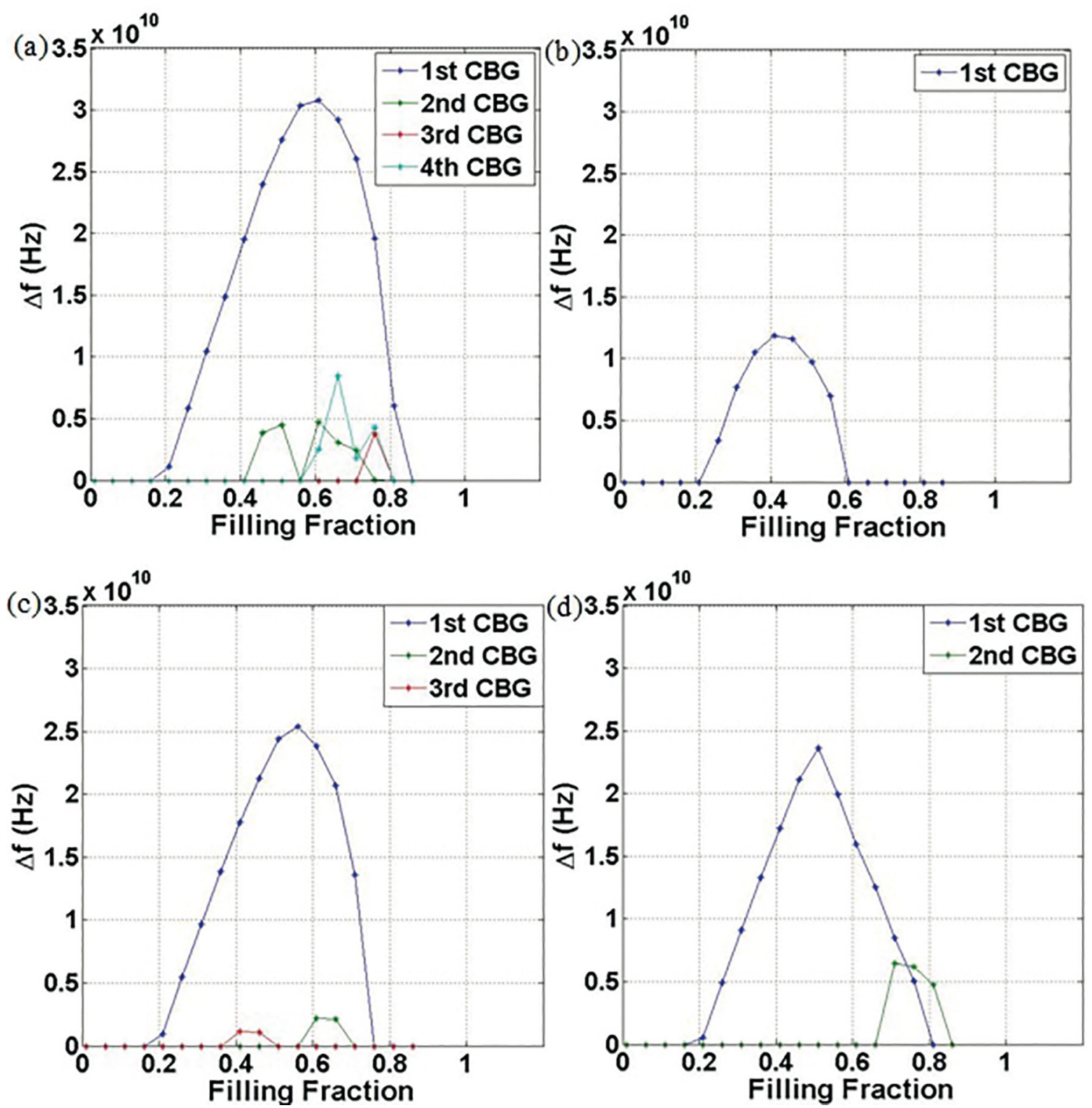

Figure 14. Elastic complete band gap widths between $\mathrm{XY}$ and $\mathrm{Z}$ modes of $\mathrm{BaTiO}_{3}$ inclusions in a polymeric matrix as a function of filling fraction for triangular lattice. The following types of inclusions are considered - circular (a), hollow circular (b), square (c) and rotated square with a $45^{\circ}$ angle of rotation with respect to the $x, y$ axes. 

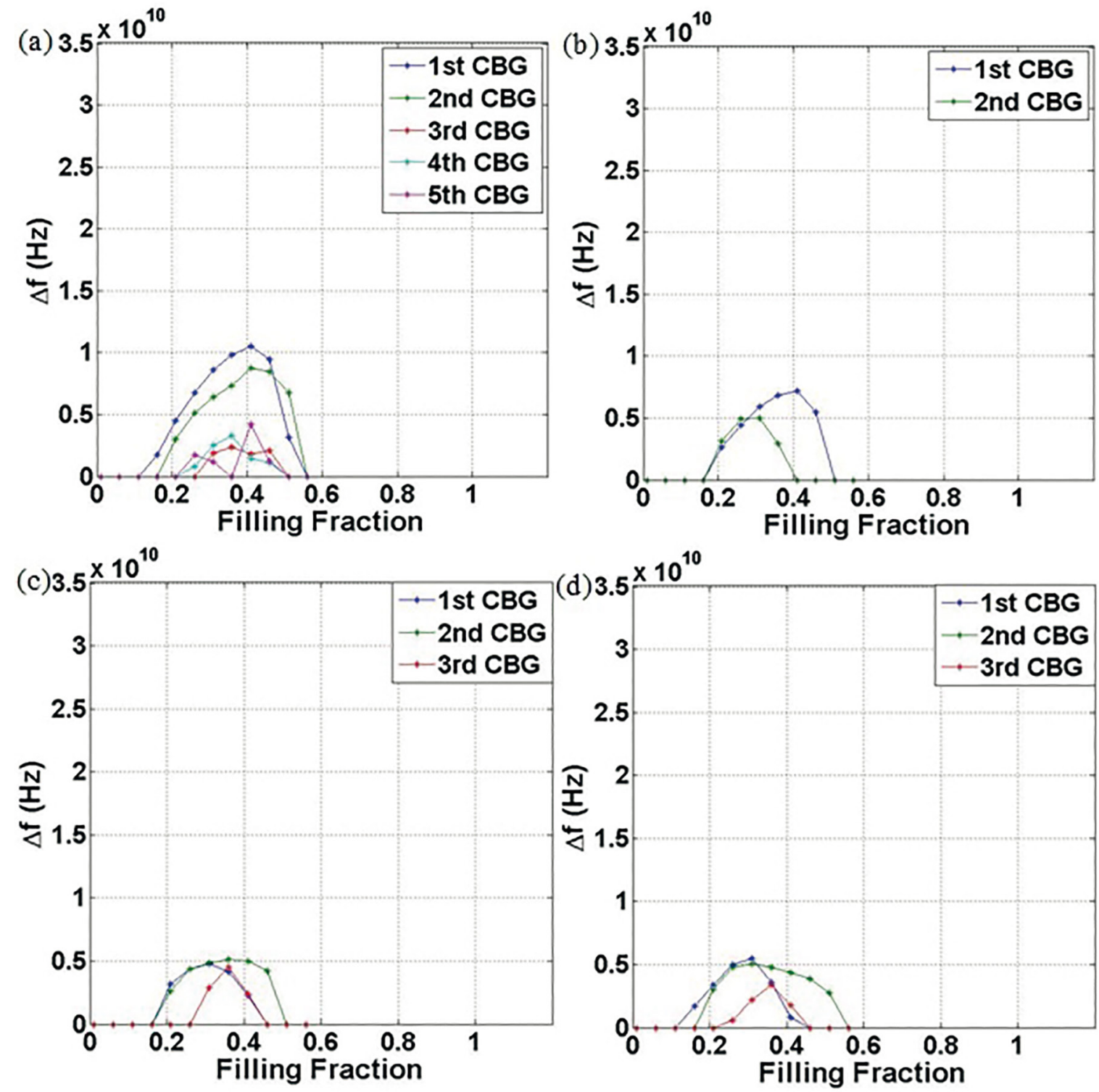

Figure 15. Elastic complete band gap widths between $\mathrm{XY}$ and $\mathrm{Z}$ modes of $\mathrm{BaTiO}_{3}$ inclusions in a polymeric matrix as a function of filling fraction for honeycomb lattice. The following types of inclusions are considered - circular (a), hollow circular (b), square (c) and rotated square with a $45^{\circ}$ angle of rotation with respect to the $x, y$ axes.

\section{Conclusion}

Broad complete band gaps are obtained for a nanopiezoelectric $\mathrm{PC}$, consisting of $\mathrm{BaTiO}_{3}$ inclusions embedded in a polymeric matrix, for different inclusion geometries and different lattices. We firstly study the elastic band structure for a fixed filling fraction of 0.335 considering $X Y$ and $Z$ modes. For square lattice, the broadest complete band gap is found for rotated square inclusion with $11.97 \mathrm{GHz}$ of bandwidth. This inclusion geometry is also the best one for rectangular lattice. Triangular lattice presents the broadest complete band gap than the other lattices for circular inclusion. The elastic band structure for honeycomb and Kagomé lattices presents several complete band gaps. However, for Kagomé lattice, all of them are narrower than the complete band gaps from square, rectangular, triangular and honeycomb lattices.

The $\mathrm{Z}$ mode with and without piezoelectric effect is also investigated for a fixed filling fraction of 0.335 . This effect is significant on the band gaps for all lattices and inclusions, and it is more evident in higher frequencies for circular, square and rotated square inclusions. However, piezoelectricity is significant in lower frequencies for hollow circular inclusion.

We also analyze the complete band gap bandwidths as a function of filling fraction for all inclusions and lattices with piezoelectric effect. Among square, rectangular and triangular lattices, triangular lattice presents the best performance for circular inclusion (first complete band gap), hollow circular 

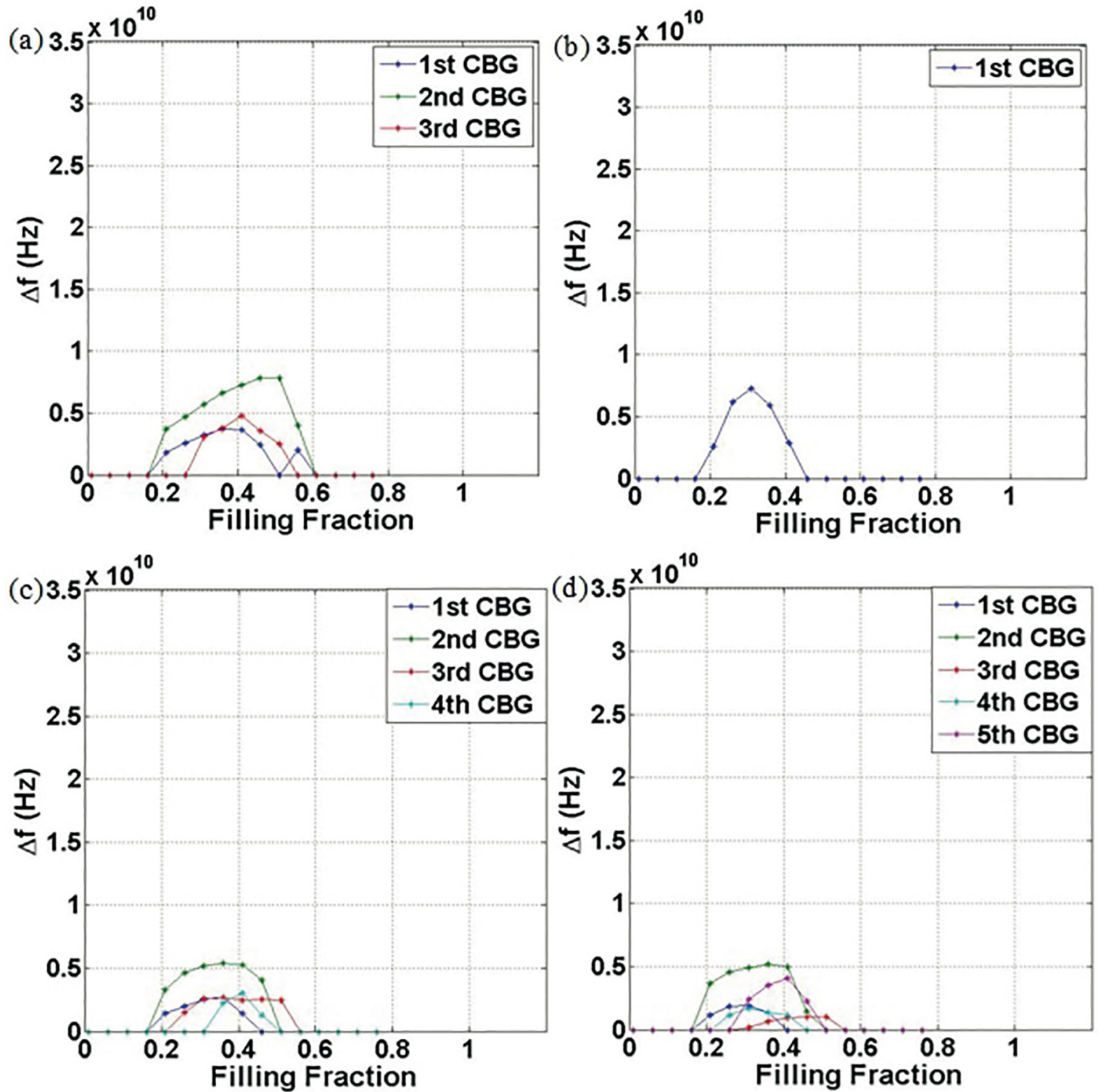

Figure 16. Elastic complete band gap widths between $\mathrm{XY}$ and $\mathrm{Z}$ modes of $\mathrm{BaTiO}_{3}$ inclusions in a polymeric matrix as a function of filling fraction for Kagomé lattice. The following types of inclusions are considered - circular (a), hollow circular (b), square (c) and rotated square with a $45^{\circ}$ angle of rotation with respect to the $x, y$ axes.

and rotated square inclusions. For square inclusion, square lattice shows the best performance. Among honeycomb and Kagomé lattices, the best performance is found for honeycomb lattice considering circular, hollow circular and rotated square inclusions.

The best band gap performance (i.e. highest band gap width and band gap opened up in a broad range of filling fraction) of the nano-piezoelectric PC investigated is for circular inclusion in a triangular lattice. However, it also depends on the application, for instance the frequency range of interest. Finally, we suggest that elastic complete band gaps in nano-piezoelectric PCs enlarge the potential applications for elastic vibration control in $\mathrm{GHz}$.

\section{Acknowledgments}

The authors gratefully acknowledge the financial support of this investigation by the Brazilian research funding agency FAPEMA (State Funding Agency of Maranhão) and by IFMA (Federal Institute of Education, Science and Technology of Maranhão).

\section{References}

1. Sigalas M, Economou EN. Band structure of elastic waves in two dimensional systems. Solid State Communications. 1993;86(3):141-143. 
2. Kushwaha MS, Halevi P, Martínez G, Dobrzynski L, DjafariRouhani B. Theory of acoustic band structure of periodic elastic composites. Physical Review B. 1994;49(4):2313-2322.

3. Huang GL, Sun CT. Modeling heterostructures of nanophononic crystals by continuum model with microstructures. Applied Physics Letters. 2006;88(26):261908.

4. Hepplestone SP, Srivastava GP. Hypersonic modes in nanophononic semiconductors. Physical Review Letters. 2008;101(10):105502.

5. Schneider D, Liaqat F, El Boudouti EH, El Hassouani Y, Djafari-Rouhani B, Tremel W, et al. Engineering the hypersonic phononic band gap of hybrid Bragg stacks. Nano Letters. 2012;12(6):3101-3108

6. Graczykowski B, Sledzinska M, Kehagias N, Alzina F, Reparaz JS, Sotomayor Torres CM. Hypersonic phonon propagation in one-dimensional surface phononic crystal. Applied Physics Letters. 2014;104(12):123108.

7. Pennec Y, Vasseur JO, Djafari-Rouhani B, Dobrzynski L, Deymier PA. Two-dimensional phononic crystals: Examples and applications. Surface Science Reports. 2010;65(8):229-291.

8. Huang J, Shi Z. Attenuation zones of periodic pile barriers and its application in vibration reduction for plane waves. Journal of Sound and Vibration. 2013;332(19):4423-4439.

9. Yu K, Chen T, Wang X. Band gaps in the low-frequency range based on the two-dimensional phononic crystal plates composed of rubber matrix with periodic steel stubs. Physica B: Condensed Matter. 2013;416:12-16.

10. Anjos V, Arantes A. Phononic band structure in carbon microtube composites. RSC Advances. 2015;5(15):11248-11253.

11. Miranda EJP Jr, Dos Santos JMC. Flexural wave band gaps in metamaterial elastic beam. In: Proceedings of the $23^{\text {rd }} A B C M$ International Congress of Mechanical Engineering. (COBEM 2015); 2015 Dec 6-11; Rio de Janeiro, RJ, Brazil. 8 p.

12. Miranda EJP Jr, Dos Santos JMC. Phononic band gaps in $\mathrm{Al}_{2} \mathrm{O}_{3}$ / epoxy composite. Materials Science Forum. 2018;912:112-117.

13. Miranda EJP Jr, Dos Santos JMC. Flexural wave band gaps in $\mathrm{Al}_{2} \mathrm{O}_{3}$ /epoxy composite rectangular plate using Mindlin theory. In: Proceedings of the $3^{\text {rd }}$ Brazilian Conference on Composite Materials (BCCM-3); 2016 Aug 28-31; Gramado, RS, Brazil. $8 \mathrm{p}$.

14. Miranda EJP Jr, Dos Santos JMC. Elastic wave band gaps in a two-dimensional magnetoelectroelastic phononic crystal. Revista Interdisciplinar de Pesquisa em Engenharia. 2016;2(13):13-26.

15. Yablonovitch E. Inhibited spontaneous emission in solid-state physics and electronics. Physical Review Letters. 1987;58(20):20592062 .

16. John S. Strong localization of photons in certain disordered dielectric superlattices. Physical Review Letters. 1987;58(23):24862489 .

17. Olsson III RH, El-Kady I. Microfabricated phononic crystal devices and applications. Measurement Science and Technology. 2009;20:012002.

18. Jensen JS. Phononic band gaps and vibrations in one- and two-dimensional mass-spring structures. Journal of Sound and Vibration. 2003;266(5):1053-1078.
19. Wang G, Wen JH, Wen XS. Quasi-one-dimensional phononic crystals studied using the improved lumped-mass method: Application to locally resonant beams with flexural wave band gap. Physical Review B. 2005;71(10):104302.

20. Casadei F, Beck BS, Cunefare KA, Ruzzene M. Vibration control of plates through hybrid configurations of periodic piezoeletric shunts. Journal of Intelligent Material Systems and Structures. 2012;23(10):1169-1177.

21. Miranda EJP Jr, Dos Santos JMC. Flexural wave band gaps in elastic metamaterial thin plate. In: Proceedings of the $I X$ Mechanical Engineering Brazilian Congress (CONEM 2016); 2016 Aug 21-25; Fortaleza, CE, Brazil; 10 p.

22. Miranda EJP Jr, Dos Santos JMC. Flexural wave band gaps in elastic metamaterial beam. In: Proceedings of the International Conference on Noise and Vibration Engineering (ISMA2016). 2016 Sep 19-23; Leuven, Belgium. p. 2099-2113.

23. Ho KM, Cheng CK, Yang Z, Zhang XX, Sheng P. Broadband locally resonant sonic shields. Applied Physics Letters. 2003;83(26):5566.

24. Qiu C, Liu Z, Mei J, Shi J. Mode-selecting acoustic filter by using resonant tunneling of two-dimensional double phononic crystals. Applied Physics Letters. 2005;87(10):104101.

25. Yang Z, Dai HM, Chan NH, Ma GC, Sheng P. Acoustic metamaterial panels for sound attenuation in the $50-1000 \mathrm{~Hz}$ regime. Applied Physics Letters. 2010;96(4):041906.

26. Casadei F, Dozio L, Ruzzene M, Cunefare KA. Periodic shunts arrays for the control of noise radiation in an enclosure. Journal of Sound and Vibration. 2010;329(18):3632-3646.

27. Xiao Y, Wen J, Wen X. Sound transmission loss of metamaterialbased thin plates with multiple subwavelength arrays of attached resonators. Journal of Sound and Vibration. 2012;331(25):54085423.

28. Benchabane S, Khelif A, Robert L, Rauch JY, Pastureaud T, Laude V. Elastic band gaps for surface modes in an ultrasonic lithium niobate phononic crystal. In: Proceedings of SPIE Photonics Europe; 2006; Strasbourg, France. 2006. 618216.

29. Comerio MC. Can buildings be made earthquake-safe? Science. 2006;312(5771):204-206

30. Gorishnyy T, Ullal CK, Maldovan M, Fytas G, Thomas EL. Hypersonic phononic crystals. Physical Review Letters. 2005;94(11):115501.

31. Cheng W, Sainidou R, Burgardt P, Stefanou N, Kiyanova A, Efremov M, et al. Elastic properties and glass transition of supported polymer thin films. Macromolecules. 2007;40(20):72837290 .

32. Liu Z, Zhang X, Mao Y, Zhu YY, Yang Z, Chan CT, et al. Locally resonant sonic materials. Science. 2000;289(5485):1734-1736.

33. Davis BL, Hussein M. Nanophononic metamaterial: Thermal conductivity reduction by local resonance. Physical Review Letters. 2014;112(5-7):055505.

34. Wagner MR, Graczykowski B, Reparaz JS, Sachat AE, Sledzinska M, Alzina F, et al. Two-dimensional phononic crystals: Disorder matters. Nano Letters. 2016;16(9):5661-5668. 
35. Yang L, Yang N, Li B. Extreme low thermal conductivity in nanoscale 3D Si phononic crystal with spherical pores. Nano Letters. 2014;14(4):1734-1738.

36. Zen N, Puurtinen TA, Isotalo TJ, Chaudhuri S, Maasilta IJ. Engineering thermal conductance using a two-dimensional phononic crystal. Nature Communications. 2014;5:3435.

37. Anufriev R, Nomura M. Thermal conductance boost in phononic crystal nanostructures. Physical Review B. 2015;91:245417.

38. Lacatena V, Haras M, Robillard JF, Monfray S, Skotnicki T, Dubois E. Toward quantitative modeling of silicon phononic thermocrystals. Applied Physics Letters. 2015;106(11):114104.

39. Honarvar H, Yang L, Hussein MI. Thermal transport size effects in silicon membranes featuring nanopillars as local resonators. Applied Physics Letters. 2016; 108(263101):1-5.

40. Wilm M, Ballandras S, Laude V, Pastureaud T, Pierre G, Steichen W. A plane-wave-expansion approach for modelling acoustic propagation in 2D and 3D piezoelectric periodic structures. In: IEEE Ultrasonics Symposium; 2001 Oct 7-10; Atlanta, GA, USA. p. 977-980.

41. Wilm M, Ballandras S, Laude V, Pastureaud T. A full 3D planewave-expansion model for 1-3 piezoelectric composite structures. Journal of the Acoustical Society of America. 2002;112(3 Pt 1):943-952.

42. Wilm M, Khelif A, Ballandras S, Laude V, Djafari-Rouhani B. Out-of-plane propagation of elastic waves in two-dimensional phononic band-gap materials. Physical Review E. 2003;67(6):065602.

43. Hou Z, Wu F, Liu Y. Phononic crystals containing piezoelectric material. Solid State Communications. 2004;130(11):745-749.

44. Wu TT, Hsu ZC, Huang ZG. Band gaps and the electromechanical coupling coefficient of a surface acoustic wave in a twodimensional piezoelectric phononic crystal. Physical Review B. 2005;71(6):064303.

45. Hsu JC, Wu TT. Bleustein-Gulyaev-Shimizu acoustic waves in two-dimensional piezoelectric phononic crystals. IEEE Transactions on Ultrasonics, Ferroelectrics, and Frequency Control. 2006;53(6):1169-1176.

46. Wang YZ, Li FM, Huang WH, Wang YS. Effects of inclusion shapes on the band gaps in two-dimensional piezoelectric phononic crystals. Journal of Physics: Condensed Matter. 2007;19(49):496204.

47. Hsu JC, Wu TT. Calculations of Lamb wave band gaps and dispersions for piezoelectric phononic plates using Mindlin's theory-based plane wave expansion method. IEEE Transactions on Ultrasonics, Ferroelectrics, and Frequency Control. 2008;55(2):431-441.

48. Qian ZH, Jin F, Li FM, Kishimoto K. Complete band gaps in two-dimensional piezoelectric phononic crystals with $\{1-$ 3 \} connectivity family. International Journal of Solids and Structures. 2008;45(17):4748-4755.

49. Zou XY, Chen Q, Liang B, Cheng JC. Control of the elastic wave bandgaps in two-dimensional piezoelectric periodic structures. Smart Materials and Structures. 2008;17(1):015008.

50. Wang YZ, Li FM, Kishimoto K, Wang YW, Huang WH. Wave band gaps in three-dimensional periodic piezoelectric structures. Mechanics Research Communications. 2009;36(4):461-468.
51. Wang Y, Li F, Wang Y, Kishimoto K, Huang W. Tuning of band gaps for a two-dimensional piezoelectric phononic crystal with a rectangular lattice. Acta Mechanica Sinica. 2009;25(1):65-71.

52. Huang Y, Zhang CL, Chen WQ. Elastic wave band structures and defect states in a periodically corrugated piezoelectric plate. Journal of Applied Mechanics. 2014;81(8):081005.

53. Lian Z, Jiang S, Hu H, Dai L, Chen X, Jiang W. An enhanced plane wave expansion method to solve piezoelectric phononic crystal with resonant shunting circuits. Shock and Vibration. 2016;2016:4015363.

54. Li L, Guo Y. Analysis of longitudinal waves in rod-type piezoelectric phononic crystals. Crystals. 2016;6(4):45.

55. Robillard JF, Bou Matar O, Vasseur JO, Deymier PA, Stippinger M, Hladky-Hennion AC, et al. Tunable magnetoelastic phononic crystals. Applied Physics Letters. 2016;95(12):124104.

56. Vasseur JO, Bou Matar O, Robillard JF, Hladky-Hennion AC, Deymier PA. Band structures tunability of bulk 2D phononic crystals made of magneto-elastic materials. AIP Advances. 2011;1(4):041904.

57. Bou Matar O, Robillard JF, Vasseur JO, Hladky-Hennion AC, Deymier PA, Preobrazhensky V. Band gap tunability of magneto-elastic phononic crystal. Journal of Applied Physics. 2012;111(5):054901.

58. Zhou C, Sai Y, Chen J. Tunable Lamb wave band gaps in twodimensional magnetoelastic phononic crystal slabs by an applied external magnetostatic field. Ultrasonics. 2016;71:69-74.

59. Wang YZ, Li FM, Huang WH, Jiang X, Wang YS, Kishimoto K. Wave band gaps in two-dimensional piezoelectric/piezomagnetic phononic crystals. International Journal of Solids and Structures. 2008;48(14-15):4203-4210.

60. Wang YZ, Li FM, Kishimoto K, Wang YS, Huang WH. Elastic wave band gaps in magnetoelectroelastic phononic crystals. Wave Motion. 2009;46(1):47-56.

61. Wang YZ, Li FM, Kishimoto K, Wang YS, Huang WH. Band gap behaviours of periodic magnetoelectroelastic composite structures with kagome lattices. Waves in Random and Complex Media. 2009;19(3):509-520.

62. Lan M, Wei P. Band gap of piezoelectric/piezomagnetic phononic crystal with graded interlayer. Acta Mechanica. 2014;225(6):1779-1794

63. Guo X, Wei P, Lan M, Li L. Dispersion relations of elastic waves in one-dimensional piezoelectric/piezomagnetic phononic crystal with functionally graded interlayers. Ultrasonics. 2016;70:158 171.

64. El-Kady I, Olsson III RH, Fleming JG. Phononic band-gap crystals for radio frequency communications. Applied Physics Letters. 2008;92(23):233504.

65. Soliman YM, Su MF, Leseman ZC, Reinke CM, El-Kady I, Olsson III RH. Phononic crystals operating in the gigahertz range with extremely wide band gaps. Applied Physics Letters. 2010;97(19):193502.

66. Robillard JF, Devos A, Roch-Jeune I. Time-resolved vibrations of two-dimensional hypersonic phononic crystals. Physical Review B. 2007;76(9):092301. 
67. Ezzahri Y, Grauby S, Rampnoux JM, Michel H, Pernot G, Claeys W, et al. Coherent phonons in $\mathrm{Si} / \mathrm{Si}$ Ge superlattices. Physical Review B. 2007;75(19):195309.

68. Olsson III RH, Ziaei-Moayyed M, Kim B, Reinke C, Su MF, Hopkins $\mathrm{P}$, et al. Micro and nano fabricated phononic crystals: Technology and applications. In: Proceedings of the IEEE International Ultrasonics Symposium; 2011 Oct 18-21; Orlando, FL, USA. p. 983-988.

69. Graczykowski B, Sledzinska M, Alzina F, Gomis-Bresco J, Reparaz JS, Wagner MR, et al. Phonon dispersion in hypersonic two-dimensional phononic crystal membranes. Physical Review B. 2015;91(7):075414.

70. Travagliati M, Nardi D, Giannetti C, Gusev V, Pingue P, Piazza $\mathrm{V}$, et al. Interface nano-confined acoustic waves in polymeric surface phononic crystals. Applied Physics Letters. 2015;106(2):021906.

71. Sadat SM, Wang RY. Colloidal nanocrystal superlattices as phononic crystals: Plane wave expansion modeling of phonon band structure. RSC Advances. 2016;6(50):44578-44587.

72. Sgouros AP, Neupane MR, Sigalas MM, Aravantinos-Zafiris N, Lake RK. Nanoscale phononic interconnects in $\mathrm{THz}$ frequencies. Physical Chemistry Chemical Physics. 2014;16(42):2335523364.

73. Zhen N, Wang YS, Zhang C. Surface/interface effect on band structures of nanosized phononic crystals. Mechanics Research Communications. 2012;46:81-89.

74. Zhen N, Wang YS, Zhang C. Bandgap calculation of in-plane waves in nanoscale phononic crystals taking in account surface/ interface effects. Physica E: Low-dimensional Systems and Nanostructures. 2013;54:125-132.
75. Abdollahi A, Moravvej-Farshi MK. Designing a hypersonic filter on a nanophononic crystal platform. In: Proceedings of the $22^{\text {nd }}$ Iranian Conference on Electrical Engineering (ICEE 2014); 2014 May 20-22; Tehran, Iran. p. 26-28.

76. Wang ZL, Song J. Piezoelectric nanogenerators based on zinc oxide nanowire arrays. Science. 2006;312(5771):242-246.

77. Wang ZL. The new field of nanopiezotronics. Materialstoday. 2007;10(5):20-28

78. Yang F, Li JCM. Micro and Nano Mechanical Testing of Materials and Devices. New York: Springer; 2008.

79. Wang X. Piezoelectric nanogenerators - Harvesting ambient mechanical energy at the nanometer scale. Nano Energy. 2012;1(1):13-24.

80. Brillouin L. Wave Propagation in Periodic Structures. Mineola: Dover Publications; 1946.

81. Kuang W, Hou Z, Liu Y. The effects of shapes and symmetries of scatterers on the phononic band gap in 2D phononic crystals. Physics Letters A. 2004;332(5-6):481-490.

82. Floquet $\mathrm{G}$. Sur les équations différentielles linéaires à coefficients périodiques. Annales Scientifiques de L'École Normale Supérieure. $1883 ; 12: 47-88$.

83. Bloch F. Über die quantenmechanik der electron in kristallgittern. Zeitschrift für Physik. 1929;52(7-8):555-600.

84. Cassagne D, Jouanin C, Bertho D. Hexagonal photonic-band gap structures. Physical Review B. 1996;53(11):7134-7142.

85. Gao Z, Fang J, Zhang Y, Jiang L. Band structure research of a $2 \mathrm{D}$ honeycomb lattice phononic crystal. International Journal of Electrochemical Science. 2013;8:7918-7925. 